\title{
Heavy $Z^{\prime}$ bosons in the secluded $U(1)^{\prime}$ model at hadron colliders
}

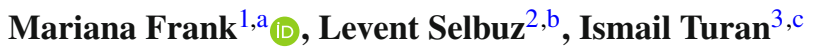 \\ ${ }^{1}$ Department of Physics, Concordia University, 7141 Sherbrooke St. West, Montreal, QC H4B 1R6, Canada \\ ${ }^{2}$ Department of Engineering Physics, Ankara University, 06100 Ankara, Turkey \\ ${ }^{3}$ Department of Physics, Middle East Technical University, 06800 Ankara, Turkey
}

Received: 1 October 2020 / Accepted: 10 May 2021 / Published online: 27 May 2021

(C) The Author(s) 2021

\begin{abstract}
We study $Z^{\prime}$ phenomenology at hadron colliders in an $U(1)^{\prime}$ extended MSSM. We choose a $U(1)^{\prime}$ model with a secluded sector, where the tension between the electroweak scale and developing a large enough mass for $Z^{\prime}$ is resolved by incorporating three additional singlet superfields into the model. We perform a detailed analysis of the production, followed by decays, including into supersymmetric particles, of a $Z^{\prime}$ boson with mass between 4 and $5.2 \mathrm{TeV}$, with particular emphasis on its possible discovery. We select three different scenarios consistent with the latest available experimental data and relic density constraints, and concentrate on final signals with $2 \ell+E_{T}, 4 \ell+E_{T}$ and $6 \ell+E_{T}$. Including the SM background from processes with two, three or four vector bosons, we show the likelihood of observing a $Z^{\prime}$ boson is not promising for the HL-LHC at $14 \mathrm{TeV}$. While at 27 and $100 \mathrm{TeV}$, the situation is more optimistic, and we devise specific benchmark scenarios which could be observed.
\end{abstract}

\section{Introduction}

After the discovery of the Higgs boson, the missing piece of the Standard Model (SM), the quest for physics beyond the SM (BSM) has intensified, both from theorists and experimentalists. The searches and analyses are motivated by the fact that while precise theoretical calculations within the SM have been confirmed by a wide range of experimental data establishing SM as a well-tested physics theory, it still lacks explanation for some of the fundamental phenomena, such as matter-antimatter asymmetry of the universe, dark matter, or neutrino oscillations. It is also plagued by theoretical inconsistencies, so it is at best incomplete (for instance, it does not include gravity). In fact, the discovery of the Higgs boson, with mass of the order of the electroweak scale, as expected,

\footnotetext{
a e-mail: mariana.frank@ concordia.ca (corresponding author)

b e-mail: Levent.Selbuz@eng.ankara.edu.tr

c e-mail: ituran@metu.edu.tr
}

points towards a higher structure, because in the SM, it is unclear why the Higgs boson is so much lighter than the Planck mass, as one expects that the large quantum contributions to the square of the Higgs boson mass would inevitably make the mass huge, and comparable to the scale at which new physics appears, unless there is an incredible amount of fine-tuning cancellation between the quadratic radiative corrections and the bare mass. One can explore BSM physics taking a model independent approach, by assuming an effective field theoretic approach [1], which provides a general framework where higher order interactions of independent operators are built and one would be able to match them to explicit ultraviolet complete models in a systematic way. Or, one can enlarge the particle and/or gauge symmetry of the model.

Of the latter, the addition of supersymmetry (SUSY) to the SM is the most popular BSM scenario. It resolves the Higgs mass/gauge hierarchy problem, and provides, in its simplest scenario, the Minimal Supersymmetric Standard Model (MSSM), a natural dark matter (DM) candidate. It does not, however, explain neutrino masses and it provides no resolution for the $\mu$ problem [2-5]. The $\mu$ parameter, so-called higgsino mass term, enters the supersymmetric Lagrangian as $\mu \hat{H}_{u} \hat{H}_{d}$, to give masses to the fermionic components of the Higgs bosons, and thus it is expected to be of the order of the SUSY-breaking scale. But the $\mu$ term also enters in the scalar potential, so, for successful electroweak symmetry breaking, its value should be at electroweak scale. Adding an $U(1)^{\prime}$ gauge group to the SM/MSSM symmetry group resolves this inconsistency. An additional singlet Higgs field $S$ develops a vacuum expectation value (VEV) which breaks the $U(1)^{\prime}$ symmetry and generates the $\mu$ term dynamically, with $\mu \sim \mathcal{O}(\langle S\rangle)$. In addition, the model contains three singlet right-handed neutrinos that yield masses for the lefthanded neutrinos (Dirac or Majorana).

Models with additional $U(1)^{\prime}$ groups extend the spectrum of MSSM minimally: in addition to the right-handed neu- 
trino and Higgs field $S$, they include another neutral gauge boson $Z^{\prime}$ (as well as theirs supersymmetric partners). This gauge field, a consequence of the additional $U(1)^{\prime}$ group, is predicted by many extensions of the SM. String-inspired models [2,6] and grand-unification (GUT) models usually contain a number of extra $U(1)$ symmetries. The GUT group $S O$ (10) [7] and exceptional group $E_{6}$ [8-10] are some examples. Here the $U(1)^{\prime}$ symmetries are broken at some intermediate energy scales [11], between the GUT and electroweak scales. Phenomenologically, the most interesting option is the breaking around $\mathrm{TeV}$ scales, giving rise to extra neutral $Z^{\prime}$ gauge bosons observable at colliders.

The physics of $Z^{\prime}$ bosons has been extensively studied in the literature, in models without supersymmetry [12-41], or with [5,42-53]. The additional neutral gauge bosons have received significant attention from the experimentalists, and have been searched for extensively at the LHC, in dilepton channels [54], dijet channels [55,56], di-tau [57], or $t \bar{t}$ decays [58]. Mass limits of $4 \mathrm{TeV}$ or above, depending of the particular $U(1)^{\prime}$ scenario chosen, have hindered extensive analyses of their implications for phenomenology, as the prospects of observing them at the LHC do not appear to be promising.

In supersymmetric $U(1)^{\prime}$ models, an additional problem arises. The mass of the $Z^{\prime}$ boson is, as usual, proportional to the VEV of the singlet Higgs boson $S$. But this parameter also determines the scale of the chargino/neutralino sector, thus a heavy $Z^{\prime}$ implies a heavy electroweakino sector, reducing further the interest in such models at the LHC. To avoid this link, we work in a secluded scenario [59,60], where the scalar sector of the $U(1)^{\prime}$ model is augmented by three additional singlet superfields ${ }^{1}$, whose role is to decouple the mass of the $Z^{\prime}$ from the scale of chargino and neutralino masses. The disadvantage is extending the particle spectrum of the SM more than the conventional $U(1)^{\prime}$ model, while the gain is being able to preserve a large $Z^{\prime}$ mass while allowing light charginos/neutralinos, and in particular, a light dark matter candidate, which is the lightest supersymmetric particle (LSP). ${ }^{2}$ In the secluded model, $Z^{\prime}$ decays into (light) chargino and neutralino pairs, or into sfermions can be significant, and affect the mass limits, albeit slightly.

There have been studies of the $Z^{\prime}$ boson where the mass constraints were considerably reduced, assuming the model to be leptophobic [61-63] or quark-phobic [64]. For these, $Z^{\prime}$ couplings to the leptons or quarks are tuned (by assuming family non-universality [65], by using a specific value of the kinetic mixing [63], or by a choice of $U(1)^{\prime}$ charges [65]). We propose here take a different point of view. We revisit the $U(1)^{\prime}$ model with a secluded sector, and allow for heavy

\footnotetext{
1 While only one additional singlet superfield would split the mass scales, three are needed for anomaly cancellation.

2 The LSP can be the lightest neutralino or the lightest right-handed sneutrino.
}

$Z^{\prime}$ bosons, satisfying mass limit restrictions from LHC. We concentrate on its production, decays and observability at high-luminosity (HL), and/or high-energy (HE) frontier of a future LHC, as well as the hadronic mode of a Future Circular Collider (FCC-hh).

A heavy gauge boson, with mass $M_{Z^{\prime}}>4 \mathrm{TeV}$ cannot always be treated as a narrow width, as the ratio $\Gamma_{Z^{\prime}} / M_{Z^{\prime}}$ could be $>10 \%$. In this case, interference effects are important [66-68] and must be included. New physics contributions to the $Z^{\prime}$ width may significantly decrease the branching ratios into SM particles, and therefore the mass limits quoted by the experiments may have to be revisited. Furthermore, $Z^{\prime}$ decays into supersymmetric particles represent an excellent tool to investigate the electroweak interactions at the LHC in a phase-space corner that cannot be explored by employing the usual techniques. The secluded $U(1)^{\prime}$ model is ideally suited for this analysis, as it allows the electroweakinos to be light. Therefore, the possible discovery of supersymmetry in $Z^{\prime}$ mediated processes would help to understand the role of $Z^{\prime}$ in the SUSY breaking and open the path to additional investigations, since one would need to formulate a consistent scenario accommodating both sparticles and heavy gauge bosons.

The scope of this paper is indeed the investigation of the phenomenology of $Z^{\prime}$ bosons at the LHC, assuming that they are heavy, and that they can decay into both SM and supersymmetric particles. We will analyze the decay channels of the $Z^{\prime}$ boson, including decays to neutralinos, charginos, sleptons and Higgs bosons, which are normally neglected.

In our study, we will allow the $U(1)^{\prime}$ parameters to run within suitable ranges, taking into account the recent experimental limits. Throughout this work, we will focus especially on the decay of the $Z^{\prime}$ into slepton, chargino and neutralino pairs, eventually leading to multilepton final states $2 \ell+\mathbb{E}_{T}$ or $4 \ell+E_{T}$ or $6 \ell+E_{T}$. To test the observability of such signals at the LHC, we devise benchmark scenarios and test their features at high integrated luminosity and at the center-ofmass $\sqrt{s}=14,27$ and $100 \mathrm{TeV}$. We include calculations of he $\mathrm{SM}$ background from $V V, V V V$, and $V V V V$ processes and we present a simulation analysis for the HL-LHC and future hadron colliders, indicating the significance of each scenario, and most promising observable for each signal.

Our paper is organized as follows. In Sect. 2 we describe the secluded $U(1)^{\prime}$ model with particular emphasis on its neutral gauge (in Sect. 2.1) and neutralino (in Sect. 2.2) sectors. We then proceed to analyze the implications of the model at colliders in Sect. 3, focusing first in choosing three benchmarks, which obey experimental constraints, and which are able to reproduce the correct relic density, while maximizing $Z^{\prime}$ decays into supersymmetric particles, in Sect. 3.1. Then we proceed with the analysis of $Z^{\prime}$ production and decays. We concentrate our analysis on multilepton signals Sect. 3.2, looking at $2 \ell+\mathbb{E}_{T}$ (in Sect. 3.2.1), $4 \ell+\mathbb{E}_{T}$ (in Sect. 3.2.2), 
and $6 \ell+E_{T}$ (in Sect. 3.2.3) signals. We summarize our findings and conclude in Sect. 4.

\section{The secluded $U(1)^{\prime}$ model}

We present here the main ingredients of the secluded $U(1)^{\prime}$ model, with particular emphasis on the $Z^{\prime}$ boson. The model is based on the gauge group $S U(3)_{c} \otimes S U(2)_{L} \otimes U(1)_{Y} \otimes$ $U(1)^{\prime}$, which breaks to the SM/MSSM $S U(3)_{c} \otimes S U(2)_{L} \otimes$ $U(1)_{Y}$. The additional Abelian group introduces, in addition to the MSSM superfields, three right-handed neutrino superfields $\hat{N}_{i}^{c}$ (one for each generation), four singlet superfields $\hat{S}, \hat{S}_{1}, \hat{S}_{2}$ and $\hat{S}_{3}$ and an additional neutral gauge boson and gaugino, corresponding to the gauge sector of $U(1)^{\prime}$. While only one scalar field $S$ is needed to break the symmetry, three additional singlets $S_{1}, S_{2}$ and $S_{3}$ (the secluded sector) are introduced to split the mass scale of the additional gauge boson from that of electroweakinos.

Unfortunately, anomaly cancelation requires the presence of additional superfields (namely, the exotics $\hat{\mathcal{Q}}$ and $\hat{\mathcal{L}}$ ), with exotic quantum numbers, which are assumed to be heavy and decoupled form the rest of the spectrum. We list the superfields in the model, together with the number of generations and charge assignments under the $S U(3)_{c} \otimes S U(2)_{L} \otimes$ $U(1)_{Y} \otimes U(1)^{\prime}$ gauge group in Table 1. The superpotential in this model including the exotic fields is given by

$$
\begin{aligned}
\widehat{W}= & h_{u} \widehat{Q} \cdot \widehat{H}_{u} \widehat{U}+h_{d} \widehat{Q} \cdot \widehat{H}_{d} \widehat{D}+h_{e} \widehat{L} \cdot \widehat{H}_{d} \widehat{E}+h_{s} \widehat{S} \widehat{H}_{u} \cdot \widehat{H}_{d} \\
& +\frac{1}{M_{R}} \widehat{S}_{1} \widehat{L} \cdot \widehat{H}_{u} \mathbf{h}_{v} \widehat{N}+\bar{h}_{s} \widehat{S}_{1} \widehat{S}_{2} \widehat{S}_{3}+\sum_{i=1}^{n_{\mathcal{Q}}} h_{Q} \widehat{S} \widehat{\mathcal{Q}}_{i} \widehat{\overline{\mathcal{Q}}}_{i} \\
& +\sum_{j=1}^{n_{\mathcal{L}}} h_{L}^{j} \widehat{S}_{\mathcal{L}} \widehat{\overline{\mathcal{L}}}_{j}
\end{aligned}
$$

where the fields $\widehat{\mathcal{Q}}, \widehat{\mathcal{L}}$ are the exotics, $M_{R}$ is a large mass scale and $h_{v}$ is the Yukawa coupling responsible for generating neutrino masses. This non-renormailzable term is added in the original formulation of the model to account for Dirac neutrino masses (see, for example, [69] for the origin of the term). Thus, in this form, neutrinos are Dirac particles, whose masses imply, for the Yukawa coupling [70], $h_{v} \simeq 3 \times 10^{-13}\left(\frac{\left|m_{v}\right|^{2}}{2.8 \times 10^{-3} \mathrm{eV}^{2}}\right)^{1 / 2}$.

The effective $\mu$ term is generated dynamically as $\mu=$ $h_{S}\langle S\rangle$. The scalar potential includes the $F$-term, given by

$$
\begin{aligned}
V_{F}= & h_{s}^{2}\left(\left|H_{u}\right|^{2}\left|H_{d}\right|^{2}+|S|^{2}\left|H_{u}\right|^{2}+|S|^{2}\left|H_{d}\right|^{2}\right) \\
& +\bar{h}_{s}^{2}\left(\left|S_{1}\right|^{2}\left|S_{2}\right|^{2}+\left|S_{2}\right|^{2}\left|S_{3}\right|^{2}+\left|S_{3}\right|^{2}\left|S_{1}\right|^{2}\right),
\end{aligned}
$$

while the $D$-term scalar potential is

$$
\begin{aligned}
V_{D}= & \frac{g_{1}^{2}+g_{2}^{2}}{8}\left(\left|H_{d}\right|^{2}-\left|H_{u}\right|^{2}\right)^{2} \\
& +\frac{1}{2} g_{1}^{\prime 2}\left(Q_{S}^{\prime}|S|^{2}+Q_{H_{u}}^{\prime}\left|H_{u}\right|^{2}\right. \\
& \left.+Q_{H_{d}}^{\prime}\left|H_{d}\right|^{2}+\sum_{i=1}^{3} Q_{S_{i}}^{\prime}\left|S_{i}\right|^{2}\right)^{2},
\end{aligned}
$$

where $g_{1}, g_{2}$ and $g_{1}^{\prime}$ are the coupling constants for the $U(1)_{Y}$, $S U(2)_{L}$ and $U(1)^{\prime}$ gauge groups while $Q_{\phi}^{\prime}$ is the $U(1)^{\prime}$ charge of the field $\phi$. Finally, the potential includes the SUSYbreaking soft terms, expressed in terms of soft-SUSY breaking mass parameters $M_{i}^{2}$ and triple scalar couplings $A_{i}$ as

$$
\begin{aligned}
V_{\text {soft }}= & M_{H_{u}}^{2}\left|H_{u}\right|^{2}+M_{H_{d}}^{2}\left|H_{d}\right|^{2}+M_{S}^{2}|S|^{2}+\sum_{i=1}^{3} M_{S_{i}}^{2}\left|S_{i}\right|^{2} \\
& -\left(A_{s} h_{s} S H_{u} H_{d}+A_{\bar{s}} \bar{h}_{s} S_{1} S_{2} S_{3}+\text { h.c. }\right) \\
& +\left(M_{S S_{1}}^{2} S S_{1}+M_{S S_{2}}^{2} S S_{2}+M_{S_{1} S_{2}}^{2} S_{1}^{\dagger} S_{2}+\text { h.c. }\right) .
\end{aligned}
$$

The symmetry-breaking sector of the model is very complex, and finding an acceptable minimum of the Higgs potential, even at the tree level, is non-trivial [60]. Once a minimum is found, the mass of the lightest Higgs boson can be fine tuned to $125 \mathrm{GeV}$ by small variations in the parameter $\bar{h}_{s}$. Setting masses for the additional scalars in the $\mathrm{TeV}$ range insures that the mixing with the lightest Higgs boson is small, and thus this Higgs will obey mass [71] and signal bounds [72] consistent with the SM-like Higgs found at the LHC. Additional Higgs states, in particular the lightest pseudoscalar, being heavy, will also satisfy constraints from $B_{s} \rightarrow \mu^{+} \mu^{-}$ branching ratio [73].

The $U(1)^{\prime}$ charges of the fields satisfy conditions arising the requirement of cancellation of gauge and gravitational anomalies. For instance, the charges for Higgs fields in the model are chosen so that

$$
Q_{S}^{\prime}=-Q_{S_{1}}^{\prime}=-Q_{S_{2}}^{\prime}=Q_{S_{3}}^{\prime} / 2, \quad Q_{H_{u}}^{\prime}+Q_{H_{d}}^{\prime}+Q_{S}^{\prime}=0
$$

The $U(1)^{\prime}$ charge of the quark doublet $\widehat{Q}$ is kept as a free parameter after the normalization

$$
\begin{aligned}
& Q_{H_{u}}^{\prime}=-2, Q_{H_{d}}^{\prime}=1, Q_{S}^{\prime}=1, Q_{S_{1}}^{\prime}=-1, Q_{S_{2}}^{\prime}=-1, \\
& Q_{S_{3}}^{\prime}=2 .
\end{aligned}
$$

A complete list of conditions for anomaly cancellations in the model, and a choice of charge assignments of the SM and exotic quarks and leptons in the model can be found in [70]. 
Table 1 Superfield (SF) configuration in the secluded $U(1)^{\prime}$ model, including notations for fermionic and bosonic states, number of generations, and charges under $U(1)_{Y} \otimes S U(2)_{L} \otimes S U(3)_{C} \otimes U(1)^{\prime}$

\begin{tabular}{|c|c|c|c|c|}
\hline SF & $\operatorname{Spin} 0$ & $\operatorname{Spin} \frac{1}{2}$ & Generations & Charges \\
\hline$\hat{Q}$ & $\tilde{Q}$ & $Q$ & 3 & $\left(\frac{1}{6}, \mathbf{2}, \mathbf{3}, Q_{Q}^{\prime}\right)$ \\
\hline$\hat{L}$ & $\tilde{L}$ & $L$ & 3 & $\left(-\frac{1}{2}, \mathbf{2}, \mathbf{1}, Q_{L}^{\prime}\right)$ \\
\hline$\hat{H}_{d}$ & $H_{d}$ & $\tilde{H}_{d}$ & 1 & $\left(-\frac{1}{2}, \mathbf{2}, \mathbf{1}, Q_{H_{d}}^{\prime}\right)$ \\
\hline$\hat{H}_{u}$ & $H_{u}$ & $\tilde{H}_{u}$ & 1 & $\left(\frac{1}{2}, \mathbf{2}, \mathbf{1}, Q_{H_{u}}^{\prime}\right)$ \\
\hline$\hat{D}$ & $\tilde{D}_{R}^{*}$ & $D_{R}^{*}$ & 3 & $\left(\frac{1}{3}, \mathbf{1}, \overline{\mathbf{3}}, Q_{d}^{\prime}\right.$ \\
\hline$\hat{U}$ & $\tilde{U}_{R}^{*}$ & $U_{R}^{*}$ & 3 & $\left(-\frac{2}{3}, \mathbf{1}, \overline{\mathbf{3}}, Q_{u}^{\prime}\right)$ \\
\hline$\hat{E}$ & $\tilde{E}_{R}^{*}$ & $E_{R}^{*}$ & 3 & $\left(1, \mathbf{1}, \mathbf{1}, Q_{e}^{\prime}\right)$ \\
\hline$\hat{N}$ & $\tilde{N}^{*}$ & $N^{*}$ & 3 & $\left(0, \mathbf{1}, \mathbf{1}, Q_{v}^{\prime}\right)$ \\
\hline$\hat{S}$ & $S$ & $\tilde{S}$ & 1 & $\left(0, \mathbf{1}, \mathbf{1}, Q_{s}^{\prime}\right)$ \\
\hline$\hat{S}_{1}$ & $S_{1}$ & $\tilde{S}_{1}$ & 1 & $\left(0, \mathbf{1}, \mathbf{1}, Q_{s_{1}}^{\prime}\right)$ \\
\hline$\hat{S}_{2}$ & $S_{2}$ & $\tilde{S}_{2}$ & 1 & $\left(0, \mathbf{1}, \mathbf{1}, Q_{s_{2}}^{\prime}\right)$ \\
\hline$\hat{S}_{3}$ & $S_{3}$ & $\tilde{S}_{3}$ & 1 & $\left(0, \mathbf{1}, \mathbf{1}, Q_{s_{3}}^{\prime}\right)$ \\
\hline$\hat{\mathcal{Q}}$ & $\tilde{\mathcal{Q}}$ & $\mathcal{Q}$ & 3 & $\left(Y_{\mathcal{Q}}, \mathbf{1}, \mathbf{1}, Q_{\mathcal{Q}}^{\prime}\right)$ \\
\hline$\hat{\overline{\mathcal{Q}}}$ & $\tilde{\overline{\mathcal{Q}}}$ & $\overline{\mathcal{Q}}$ & 3 & $\left(Y_{\overline{\mathcal{Q}}}, \mathbf{1}, \mathbf{1}, Q_{\overline{\mathcal{Q}}^{\prime}}\right)$ \\
\hline$\hat{\mathcal{L}}$ & $\tilde{\mathcal{L}}$ & $\mathcal{L}$ & 2 & $\left(Y_{\mathcal{L}}, \mathbf{1}, \mathbf{1}, Q_{\mathcal{L}}^{\prime}\right)$ \\
\hline$\hat{\overline{\mathcal{L}}}$ & $\tilde{\overline{\mathcal{L}}}$ & $\overline{\mathcal{L}}$ & 2 & $\left(Y_{\overline{\mathcal{L}}}, \mathbf{1}, \mathbf{1}, Q_{\overline{\mathcal{L}}^{\prime}}\right)$ \\
\hline
\end{tabular}

\subsection{Gauge boson masses and mixing}

Through spontaneous breakdown of the group $S U(2)_{L} \otimes$ $U(1)_{Y} \otimes U(1)^{\prime}$ to $U(1)_{\mathrm{em}}$ the Higgs acquire the VEVs

$$
\left\langle H_{u}\right\rangle=\left(\begin{array}{c}
0 \\
\frac{v_{u}}{\sqrt{2}}
\end{array}\right),\left\langle H_{d}\right\rangle=\left(\begin{array}{c}
\frac{v_{d}}{\sqrt{2}} \\
0
\end{array}\right),\langle S\rangle=\frac{v_{s}}{\sqrt{2}},\left\langle S_{i}\right\rangle=\frac{v_{s_{i}}}{\sqrt{2}}
$$

Here the first two VEVs are required to break the gauge symmetries of the SM, and the third to break $U(1)^{\prime}$. After symmetry breaking, one massless state (the photon) and two massive states (the $Z_{0}$ and $Z_{0}^{\prime}$ bosons which are not yet the physical eigenstates due to a non-zero mass mixing term, to be introduced below) arise as orthonormal combinations of $W_{\mu}^{3}, Y_{\mu}$ and $Y_{\mu}^{\prime}$ gauge bosons. The $W_{\mu}^{1}$ and $W_{\mu}^{2}$ combine to form $W_{\mu}^{ \pm}$, the charged vector bosons in the model. Unlike in the MSSM, the $Z_{0}$ boson is not a physical state by itself but mixes with the $Z_{0}^{\prime}$ boson. This mass mixing term arises from the fact that the Higgs doublets $H_{u, d}$ are charged under each factor of $S U(2)_{L} \otimes U(1)_{Y} \otimes U(1)^{\prime}$, and the associated mass-squared matrix is given by

$$
M_{Z_{0} Z_{0}^{\prime}}^{2}=\left(\begin{array}{cc}
M_{Z_{0}}^{2} & \Delta^{2} \\
\Delta^{2} & M_{Z_{0}^{\prime}}^{2}
\end{array}\right)
$$

in the $\left(Z_{0 \mu}, Z_{0 \mu}^{\prime}\right)$ basis, where the matrix elements are

$$
\begin{aligned}
M_{Z_{0}}^{2} & =\frac{1}{4} g_{Z}^{2}\left(v_{u}^{2}+v_{d}^{2}\right), \\
M_{Z_{0}^{\prime}}^{2} & =g_{1}^{\prime 2}\left(Q_{H_{u}}^{\prime 2} v_{u}^{2}+Q_{H_{d}}^{\prime 2} v_{d}^{2}+Q_{S}^{\prime 2} v_{s}^{2}+\sum_{i=1}^{3} Q_{S_{i}}^{\prime 2} v_{s_{i}}^{2}\right), \\
\Delta^{2} & =\frac{1}{2} g_{Z} g_{1}^{\prime}\left(Q_{H_{u}}^{\prime} v_{u}^{2}-Q_{H_{d}}^{\prime} v_{d}^{2}\right),
\end{aligned}
$$

where $g_{Z}^{2}=g_{2}^{2}+g_{1}^{2}$. The physical neutral vector bosons, $Z, Z^{\prime}$, are obtained by diagonalizing $M_{Z_{0} Z_{0}^{\prime}}^{2}$ :

$$
\left(\begin{array}{c}
Z \\
Z^{\prime}
\end{array}\right)=\left(\begin{array}{cc}
\cos \theta_{Z_{0} Z_{0}^{\prime}} & \sin \theta_{Z_{0} Z_{0}^{\prime}} \\
-\sin \theta_{Z_{0} Z_{0}^{\prime}} & \cos \theta_{Z_{0} Z_{0}^{\prime}}
\end{array}\right)\left(\begin{array}{c}
Z_{0} \\
Z_{0}^{\prime}
\end{array}\right)
$$

where

$\theta_{Z_{0} Z_{0}^{\prime}}=-\frac{1}{2} \arctan \left(\frac{2 \Delta^{2}}{M_{Z_{0}^{\prime}}^{2}-M_{Z_{0}}^{2}}\right)$

is their mass mixing angle, and

$M_{Z, Z^{\prime}}^{2}=\frac{1}{2}\left[M_{Z_{0}^{\prime}}^{2}+M_{Z_{0}}^{2} \mp \sqrt{\left(M_{Z_{0}^{\prime}}^{2}-M_{Z_{0}}^{2}\right)^{2}+4 \Delta^{4}}\right]$

are their squared masses of the corresponding mass eigenstates. The collider searches plus various indirect observations require the $Z_{0}-Z_{0}^{\prime}$ mixing angle $\theta_{Z_{0} Z_{0}^{\prime}}$ to be at most a 
few times $10^{-3}$ [15], where unavoidable model dependence arises from $Z^{\prime}$ couplings. This bound requires either $M_{Z_{0}^{\prime}}$ to be large enough (well in the TeV range) or $\Delta^{2}$ to be sufficiently suppressed by the vacuum configuration, that is, $\tan ^{2} \beta \equiv v_{u}^{2} / v_{d}^{2} \sim Q_{H_{d}}^{\prime} / Q_{H_{u}}^{\prime}$. Which of these options is realized depends on the $U(1)^{\prime}$ charge assignments and the soft-breaking mass parameters in the Higgs sector. Having large $M_{Z_{0}^{\prime}}$ term in Eq. 6 insures a small mixing angle.

We expand more on the reason for introducing the extra scalars $S_{1}, S_{2}$, and $S_{3}$. In their absence, $M_{Z_{0}^{\prime}}$ term is equal to the one given in Eq. 7 without the summation term at the end. Hence, the mass of $Z^{\prime}$ boson will be determined by the charges under $U(1)^{\prime}$, and the vacuum expectation value of the troweakinos can remain relatively light, decoupling these two scales is desirable.

Thus, in our investigations, the mass of the $Z^{\prime}$ boson will be expected to be heavy, $M_{Z^{\prime}}>4 \mathrm{TeV}$. In this case, the mixing angle between $Z_{0}$ and $Z_{0}^{\prime}$ becomes rather small, ${ }^{3}$ and the mass eigenstates $\left(Z, Z^{\prime}\right)$ are almost identical to the the original gauge states $\left(Z_{0}, Z_{0}^{\prime}\right)$. However, despite the smallness of the mixing angle $\theta_{Z_{0} Z_{0}^{\prime}}$, we keep it in our numerical analysis.

\subsection{Neutralinos in the secluded $U(1)^{\prime}$ model}

While the chargino sector is the same as in MSSM, the neutralino content is significantly enlarged by the additional $U(1)^{\prime}$ gaugino, and the four additional singlinos $\tilde{S}, \tilde{S}_{1}, \tilde{S}_{2}$ and $\tilde{S}_{3}$. In the basis $\left\{\tilde{Y}, \tilde{W}^{3}, \tilde{H}_{d}^{0}, \tilde{H}_{u}^{0}, \tilde{S}, \tilde{Y}^{\prime}, \tilde{S}_{1}, \tilde{S}_{2}, \tilde{S}_{3}\right\}$ where $\tilde{Y}, \tilde{Y}^{\prime}$ and $\widetilde{W}^{3}$ are the neutral gauge fermions of $U(1)_{Y}, U(1)^{\prime}$ and $S U(2)_{L}$, the neutralino $9 \times 9$ mass matrix is given as

$$
\mathcal{M}_{\mathcal{N}}=\left(\begin{array}{ccccccccc}
M_{\tilde{Y}} & 0 & -M_{\tilde{Y} \tilde{H}_{d}} & M_{\tilde{Y} \tilde{H}_{u}} & 0 & M_{\tilde{Y} \tilde{Y}^{\prime}} & 0 & 0 & 0 \\
0 & M_{\tilde{W}} & M_{\tilde{W} \tilde{H}_{d}} & -M_{\tilde{W} \tilde{H}_{u}} & 0 & 0 & 0 & 0 & 0 \\
-M_{\tilde{Y} \tilde{H}_{d}} & M_{\tilde{W} \tilde{H}_{d}} & 0 & -\mu_{\mathrm{eff}} & -\mu_{H_{u}} & \mu_{H_{d}}^{\prime} & 0 & 0 & 0 \\
M_{\tilde{Y} \tilde{H}_{u}} & -M_{\tilde{W} \tilde{H}_{d}} & -\mu_{\mathrm{eff}} & 0 & -\mu_{H_{d}} & \mu_{H_{u}}^{\prime} & 0 & 0 & 0 \\
0 & 0 & -\mu_{H_{u}} & -\mu_{H_{d}} & 0 & \mu_{S}^{\prime} & 0 & 0 & 0 \\
M_{\tilde{Y} \tilde{Y} \tilde{Y}^{\prime}} & 0 & \mu_{H_{d}}^{\prime} & \mu_{H_{u}}^{\prime} & \mu_{S}^{\prime} & M_{\tilde{Y}^{\prime}} & \mu_{S_{1}}^{\prime} & \mu_{S_{2}}^{\prime} & \mu_{S_{3}}^{\prime} \\
0 & 0 & 0 & 0 & 0 & \mu_{S_{1}}^{\prime} & 0 & -\frac{\bar{h}_{s} v_{s_{3}}}{\sqrt{2}} & -\frac{\bar{h}_{s} v_{s_{2}}}{\sqrt{2}} \\
0 & 0 & 0 & 0 & 0 & \mu_{S_{2}}^{\prime} & -\frac{\bar{h}_{s} v_{s_{3}}}{\sqrt{2}} & 0 & -\frac{\bar{h}_{s} v_{s_{1}}}{\sqrt{2}} \\
0 & 0 & 0 & 0 & 0 & \mu_{S_{3}}^{\prime} & -\frac{\bar{h}_{s} v_{s_{2}}}{\sqrt{2}}-\frac{\bar{h}_{s} v_{s_{1}}}{\sqrt{2}} & 0
\end{array}\right)
$$

singlet scalar $\langle S\rangle=v_{s} / \sqrt{2}$ and thus effectively proportional to the VEV $v_{s}$. At the same time, the masses of charginos and is diagonalized by $N \mathcal{M}_{\mathcal{N}} N^{\dagger}=\operatorname{diag}\left(m_{\tilde{\chi}_{1}^{0}}, \ldots, m_{\tilde{\chi}_{0}^{0}}\right)$, $0 \leq m_{\tilde{\chi}_{1}^{0}} \leq \cdots \leq m_{\tilde{\chi}_{9}^{0}}$. The parameters introduced in the neutralino mass matrix elements in Eq. 11 are defined as

$$
\begin{array}{llll}
M_{\tilde{Y} \tilde{H}_{d}}=M_{Z_{0}} \sin \theta_{W} \cos \beta, & M_{\tilde{Y} \tilde{H}_{u}}=M_{Z_{0}} \sin \theta_{W} \sin \beta, & M_{\tilde{W} \tilde{H}_{d}}=M_{Z_{0}} \cos \theta_{W} \cos \beta, & M_{\tilde{W} \tilde{H}_{u}}=M_{Z_{0}} \cos \theta_{W} \sin \beta, \\
\mu_{H_{d}}^{\prime}=g_{1}^{\prime} Q_{H_{d}}^{\prime} v_{d}, & \mu_{H_{u}}^{\prime}=g_{1}^{\prime} Q_{H_{u}}^{\prime} v_{u}, & \mu_{S}^{\prime}=g_{1}^{\prime} Q_{S}^{\prime} v_{s}, & \mu_{S_{i}}^{\prime}=g_{1}^{\prime} Q_{S_{i}}^{\prime} v_{s_{i}}, \\
\mu_{\text {eff }}=\frac{h_{s} v_{s}}{\sqrt{2}}, & \mu_{H_{d}}=\frac{h_{s} v_{d}}{\sqrt{2}}, & \mu_{H_{u}}=\frac{h_{s} u_{u}}{\sqrt{2}} . &
\end{array}
$$

and neutralinos in the model would be proportional to the effective $\mu=h_{s} v_{s} / \sqrt{2}$, and the two scales, barring serious fine-tuning of the $h_{s}$ coupling, are connected. Introducing the extra scalars, their VEVs will be added to the contribution to the $M_{Z_{0}^{\prime}}$ term (eventually determining the mass of $Z^{\prime}$ boson), while $\mu$, determining the mass scale of charginos and neutralinos, remains unchanged. As strong constraints are imposed on the $Z^{\prime}$ boson mass at LHC, while the elec-
The gaugino mass parameters $M_{\tilde{Y}}, M_{\tilde{Y}^{\prime}}$, and $M_{\tilde{Y} \tilde{Y}^{\prime}}$ are free parameters of the model, and we introduce the ratios

$$
R_{Y Y^{\prime}}=\frac{M_{\tilde{Y} \tilde{Y}^{\prime}}}{M_{\tilde{Y}}}, R_{Y^{\prime}}=\frac{M_{\tilde{Y}^{\prime}}}{M_{\tilde{Y}}} .
$$

These parameters, representing mixing of $U(1)_{Y}$ and $U(1)^{\prime}$ gauginos, and mass parameter of the $U(1)^{\prime}$ gaugino, measured relative to the $U(1)_{Y}$ gaugino mass parameter, will be

\footnotetext{
${ }^{3}$ For our chosen benchmarks, the mixing angle $\theta_{Z_{0} Z_{0}^{\prime}}$ is of $\mathcal{O}\left(10^{-4}\right)$.
} 
Table 2 The parameters characterizing benchmarks BP1, BP2 and BP3 for the secluded $U(1)^{\prime}$ model. The values of dimensionful parameters are given in $\mathrm{GeV}$

\begin{tabular}{llll}
\hline Parameters & $B P 1$ & $B P 2$ & $B P 3$ \\
\hline$g_{1}^{\prime}$ & 0.2 & 0.12 & 0.15 \\
$\tan \beta$ & 1.345 & 1.198 & 1.175 \\
$Q_{Q}^{\prime}$ & 0.6 & 0.1 & -0.81 \\
$\mu_{\text {eff }}$ & 260 & 280 & 250 \\
$\left(h_{\nu}, h_{s}, \bar{h}_{s}\right)$ & $(1.0,0.739,0.1)$ & $(1.0,0.7235,0.1)$ & $(1.0,0.724,0.1)$ \\
$\left(A_{s}, A_{\bar{s}}\right)$ & 557.7 & $(557.7,2200)$ & $(557.7,1200)$ \\
$\left(v_{s_{1}}, v_{S_{2}}, v_{s_{3}}\right)$ & $(8675,8650,8675)$ & $(6675,15,600,14,675)$ & $(12,100,14,550,14,500)$ \\
$\left(M_{\tilde{Y}}, M_{\tilde{W}}, M_{\tilde{g}}\right)$ & $(-200,2000,2500)$ & $(-760,750,2500)$ & $(-260,300,2500)$ \\
$\left(R_{Y^{\prime}}, R_{Y Y^{\prime}}\right)$ & $(5.0,4.8)$ & $(1.0,0.01)$ & $(1.0,0.01)$ \\
$\left(M_{\tilde{v}_{e}}, M_{\tilde{v}_{\mu R}}, M_{\tilde{v}_{\tau} R}\right)$ & 500 & 3000 & 500 \\
$\left(M_{L_{1}}, M_{L_{2}}, M_{L_{3}}\right)$ & 520 & 450 & 200 \\
$\left(M_{E_{1}}, M_{E_{2}}, M_{E_{3}}\right)$ & 450 & 2125 & 1700 \\
$\left(M_{Q_{1}}, M_{Q_{2}}, M_{Q_{3}}\right)$ & $(2200,2200,2400)$ & $(2200,2200,2400)$ & $(2200,2200,2400)$ \\
$\left(M_{U_{1}}, M_{U_{2}}, M_{U_{3}}\right)$ & $(2200,2200,2500)$ & $(2300,2300,2500)$ & $(2200,2200,2500)$ \\
$\left(M_{D_{1}}, M_{D_{2}}, M_{D_{3}}\right)$ & $(2300,2300,2500)$ & $\left(-9 \times 10^{6},-9 \times 10^{6}, 0\right)$ & $(2300,2300,2500)$ \\
$\left(M_{S S_{1}}^{2}, M_{S S_{2}}^{2}, M_{S_{1} S_{2}}^{2}\right)$ & $\left(-9 \times 10^{6},-9 \times 10^{6}, 0\right)$ & $(-697.75,-959.66)$ & $\left(-9 \times 10^{6},-9 \times 10^{6}, 0\right)$ \\
$\left(A_{t}, A_{b}\right)$ & $(-697.75,-959.66)$ & $(-697.75,-959.66)$ \\
\hline
\end{tabular}

seen to be important in scanning over the parameter space, as the underlying physics will be sensitive to their variation.

\section{$3 Z^{\prime}$ boson in the $U(1)^{\prime}$ model at the current and future hadron colliders}

We now proceed to the main analysis in this work, looking at the consequences of a heavy neutral gauge boson at the collider. As we shall see, and as found before, $Z^{\prime}$ bosons satisfying all collider, cosmological and low energy constraints, do not offer promising prospects for observability at the present LHC, even operating at $3 \mathrm{ab}^{-1}$. Thus, we will also analyze the prospects of observing a signal at the $\mathrm{HE}$ LHC operating at $27 \mathrm{TeV}$ as well as at the FCC-hh. As the parameter space is large, choosing realistic benchmarks is a more transparent method to show physics results than a scan. Some previous analyses of $Z^{\prime}$ at present and future colliders exist, e.g., in [74], but in that case, the authors have considered an $E_{6}$-inspired leptophobic model, constrained to yield light $Z^{\prime}$ masses. While we share with that analysis a light chargino-neutralino sector (insured in our case, by the existence of a secluded model), ours is an analysis for signals from a heavy $Z^{\prime}$ boson scenario.

\section{1 $U(1)^{\prime}$ benchmark points and relic density}

In order to give definite predictions for the production and decay rates of the $Z^{\prime}$ boson, we scan the parameter space for benchmark scenarios to showcase the salient points of the model.

The benchmark points chosen must obey five important conditions:

- The parameters chosen had to insure the stability of the vacuum;

- The points had to satisfy relic density constraints from WMAP of cold dark matter [75] for the LSP, assumed here to be the lightest neutralino;

- The mass of the $Z^{\prime}$ boson has to satisfy mass constraints from ATLAS and CMS, as discussed in the next subsection;

- Of the parameter points satisfying the above two conditions, benchmarks were chosen to enhance the supersymmetric decay signals of the $Z^{\prime}$ boson; and

- In each scenario, the lightest Higgs boson is SM-like and has $m_{H_{1}^{0}}=125 \mathrm{GeV}$.

To analyze the model, we used CalcHEP [77], micrOMEGAs [78], PYTHIA8 [79,80], Delphes [81-83], and MadAnalysis [84] to prepare the model, calculate the mass spectrum and branching ratios, calculate the relic density, generate events and eventually carry out the simulation. Our goal was to find benchmarks that satisfy cosmological constraints on dark matter and satisfy collider constraints (the invisible width of the $Z$ boson, limits on charged sparticle masses, charginos mass, first and second-generation squark masses, lightest Higgs boson mass, $\operatorname{Br}\left(B_{s, d} \rightarrow \mu^{+} \mu^{-}\right)$, 
Table 3 The mass spectra (in $\mathrm{GeV}$ ) for the supersymmetric sector and the relic density $\Omega_{D M} h^{2}$ values of the benchmark points given in Table 2 for the Secluded $U(1)^{\prime}$

\begin{tabular}{lllll}
\hline Masses & BP1 & BP2 & BP3 & Bounds \\
\hline$m_{Z^{\prime}}$ & 4250 & 4069 & 5195 & $3900[76]$ \\
$m_{H_{i}^{0}, i=1, \ldots, 6}$ & $(125.9,543,671,1077$, & $(125.0,557,1148,2418$, & $(125.3,524,1045,1611$, & $m_{H_{1}^{0}}=125.2[76]$ \\
$m_{A_{i}^{0}, i=1, \ldots, 4}$ & $4237,17719)$ & $4171,19151)$ & $5210,22171)$ & $m_{A_{1}^{0}}>93.4[76]$ \\
$m_{\tilde{\chi}_{i}^{0}, i=1, \ldots, 5}$ & $(550,719,1012,17718)$ & $(563,592,20769,19151)$ & $(531,572,1882,22170)$ & $m_{\tilde{\chi}_{1}^{0}}>50[76]$ \\
$m_{\tilde{\chi}_{i}^{0}, i=6, \ldots, 9}$ & $(51,167,262,312,613)$ & $(48,269,328,762,763)$ & $(52,195,264,303,360)$ & - \\
$\left(m_{\tilde{\chi}_{1}^{ \pm}}, m_{\tilde{\chi}_{2}^{ \pm}}\right)$ & $(1226,2004,4222,4638)$ & $(1170,1740,4047,4237)$ & $(1036,1939,4908,5551)$ & $m_{\tilde{\chi}_{1}^{ \pm}}>103.5[76]$ \\
$m_{H^{ \pm}}$ & $(256,2004)$ & $(267,763)$ & $(192,359)$ & $m_{H^{ \pm}}>80[76]$ \\
$\left(m_{\tilde{e}_{L}}, m_{\tilde{\mu}_{L}}, m_{\tilde{\tau}_{1}}\right)$ & 540.9 & 554.9 & 522.4 & $m_{\tilde{\ell}}>(82-107)[76]$ \\
$\left(m_{\tilde{e}_{R}}, m_{\tilde{\mu}_{R}}, m_{\tilde{\tau}_{2}}\right)$ & $(503,503,457)$ & 503 & $(1412,1412,473)$ & $m_{\tilde{\ell}}>(82-107)[76]$ \\
$\left(m_{\tilde{v}_{e}}, m_{\tilde{v}_{\mu}}, m_{\tilde{v}_{\tau}}\right)$ & $(457,457,503)$ & 1850 & $(473,473,1412)$ & $m_{\tilde{v}_{\ell}}>41[76]$ \\
$\left(m_{\tilde{v}_{e R}}, m_{\tilde{v}_{\mu R}}, m_{\tilde{v}_{\tau}}\right)$ & 501 & 501 & 645 & - \\
$\Omega_{D M} h^{2}$ & 553 & 3472 & 0.119 & $0.111[75]$ \\
\hline
\end{tabular}

$\operatorname{Br}\left(B \rightarrow X_{s} \gamma\right), \Delta M_{B_{s, d}}$ and various others), as outlined in $[85,86]$, and choose those exhibiting distinct decay features, while offering some promise for collider observability.

In general, the $Z^{\prime}$ boson in this model can decay into all SM fermions, into supersymmetric particles: squark, slepton, sneutrino, neutralino, chargino, in addition to Higgs-boson pairs, $W$-boson pairs and $Z H$.

The three benchmark points, and all the parameters associated with them, are given in Table 2. We give VEVs, Yukawa couplings, trilinear couplings, mass ratios and mixings for the gauginos and soft scalar fermion mass parameters. The low value of $\tan \beta \approx 1$ is consistent with constraints from $B_{s} \rightarrow \mu^{+} \mu^{-}$branching ratio [73]. For each benchmark scenario, the mass spectra for the supersymmetric partners obtained are given in Table 3 . The mass of the additional $Z^{\prime}$ boson is $\gtrsim 4 \mathrm{TeV}$, and consistent with the ATLAS [54] analyses on $Z^{\prime}$ dilepton decays. As seen in Table 2, the VEVs of the additional scalars $\left(S_{1}, S_{2}\right.$ and $\left.S_{3}\right) v_{s_{i}}, i=1,2,3$ are mostly taken above the TeV scale so that the $Z^{\prime}$ mass bound is satisfied independent of the value of the chosen VEV of the scalar field $S$. For convenience, the parameters $\mu_{\text {eff }}$ and $h_{s}$ are taken as free parameters and the VEV of $S$ is determined using the relation

$\mu_{\mathrm{eff}}=\frac{h_{s} v_{s}}{\sqrt{2}}$.

The differences between the benchmarks are the following. In BP1, the gaugino mass parameters $M_{\tilde{Y}}(200 \mathrm{GeV}) \ll$ $M_{\tilde{W}}(2000 \mathrm{GeV}), R_{Y Y^{\prime}}=4.8$ is large, while the light (lefthanded) sneutrinos and sleptons have mass $\sim 500 \mathrm{GeV}$ and are approximately degenerate. The right-handed sneutrinos are slightly heavier. In BP2, the gauginos have intermediate mass parameters $M_{\tilde{Y}}(760 \mathrm{GeV}) \simeq M_{\tilde{W}}, R_{Y Y^{\prime}}=0.01$ is very small, while the masses of the light (left-handed) sneutrinos and light sleptons are degenerate and around 500 $\mathrm{GeV}$. The heavy ones split from the light sector significantly and can have much larger masses (up to $\sim 3500$ $\mathrm{GeV})$. In BP3, the gaugino mass parameters are both light $M_{\tilde{Y}}(260 \mathrm{GeV}) \simeq M_{\tilde{W}}, R_{Y Y^{\prime}}=0.01$ is very small, while, unlike the other two scenarios, the right-handed sneutrino masses are much lighter than those of the left-handed sneutrinos. The masses of the sleptons run in this range. The neutralino parameters affect the LSP and its composition, while the slepton and sneutrino masses affect branching ratios of $Z^{\prime}$ into sfermions.

The calculation of the relic density is performed importing the model files from CalcHEP [77] into the MicrOmegas package [78]. All the numbers obtained are within the $1 \sigma$ range of the WMAP result obtained from the Sloan Digital Sky Survey [75]

$\Omega_{D M} h^{2}=0.111_{-0.015}^{+0.011}$

The relic density of dark matter $\Omega_{\mathrm{DM}} h^{2}$ is very sensitive to the parameter $R_{Y^{\prime}}$. The value of the relic density is shown in Table 3, where we also give explicit values for masses of the physical eigenstates in the Higgs and sparticle sectors. In addition, we checked that the benchmarks satisfy low energy data. For this, various flavor observables are calculated with the help of the packages SARAH $[95,96]$ and SPheno version 4.0.4 [97,98]. The results, normalized with the corresponding SM values, are listed in Table 4 . The values are all consistent with the current available data. In the same table, we give the corrections of the secluded $U(1)^{\prime}$ to the SM values for the anomalous magnetic moments of electron, muon and tau, $\Delta a_{e}, \Delta a_{\mu}, \Delta a_{\tau}$. The measured values for the first two indicate a departure from the SM, in opposite directions 
Table 4 Leptonic anomalous moments corrections and flavor observables for each benchmark scenario considered in this study

\begin{tabular}{|c|c|c|c|c|}
\hline Observable & $B P 1$ & $B P 2$ & $B P 3$ & Bounds \\
\hline$\Delta a_{e}$ & $5.68 \times 10^{-16}$ & $1.14 \times 10^{-15}$ & $3.37 \times 10^{-16}$ & $-(8.7 \pm 3.6) \times 10^{-13}[87-89]$ \\
\hline$\Delta a_{\mu}$ & $2.43 \times 10^{-11}$ & $4.86 \times 10^{-11}$ & $1.44 \times 10^{-11}$ & $(2.7 \pm 0.9) \times 10^{-9}[90,91]$ \\
\hline$\Delta a_{\tau}$ & $7.82 \times 10^{-9}$ & $1.12 \times 10^{-8}$ & $-4.78 \times 10^{-10}$ & $\left|\Delta a_{\tau}\right|<1.75 \times 10^{-5}[92]$ \\
\hline$\frac{\operatorname{Br}\left(B \rightarrow X_{s} \gamma\right)}{\operatorname{Br}\left(B \rightarrow X_{s} \gamma\right)_{\mathrm{SM}}}$ & 1.18 & 1.17 & 1.15 & $1.05 \pm 0.11[76]$ \\
\hline$\frac{\operatorname{Br}\left(B_{s, d}^{0} \rightarrow \mu^{+} \mu^{-}\right)}{\operatorname{Br}\left(B_{s, d}^{0} \rightarrow \mu^{+} \mu^{-}\right)_{\mathrm{SM}}}$ & 1.09 & 1.11 & 1.10 & $0.83 \pm 0.25[76]$ \\
\hline$\frac{\operatorname{Br}\left(B^{+} \rightarrow \tau^{+} v_{\tau}\right)}{\operatorname{Br}\left(B^{+} \rightarrow \tau^{+} v_{\tau}\right) \mathrm{SM}}$ & 0.991 & 0.991 & 0.991 & $1.04 \pm 0.34[76]$ \\
\hline$\Delta M_{B_{(s, d)}} / \Delta M_{B_{(s, d)}}^{\mathrm{SM}}$ & $(1.10,1.04)$ & $(1.12,1.04)$ & $(1.12,1.04)$ & $(1.00 \pm 0.15,0.86 \pm 0.28)[76]$ \\
\hline$R_{K} / R_{K}^{\mathrm{SM}}$ & 1.00 & 1.00 & 1.00 & $1.00 \pm 0.17[76]$ \\
\hline$\epsilon_{K} / \epsilon_{K}^{\mathrm{SM}}$ & 1.00 & 1.00 & 1.00 & $0.99 \pm 0.18[76,93]$ \\
\hline$\Delta M_{K} / \Delta M_{K}^{\mathrm{SM}}$ & 1.00 & 1.00 & 1.00 & $1.24 \pm 0.16[76,94]^{\mathrm{a}}$ \\
\hline
\end{tabular}

In the theoretical calculation of the quoted value for $\Delta M_{K}$ ratio assumes contributions from both the so-called short distance and long distance physics. However, the latter part is not very reliable and needs improvement which might drive the value in either direction. The ratio becomes $1.12 \pm 0.44$ if only the short distance contribution is kept

for the electron [87-89] and muon [90,91]:

$$
\begin{aligned}
& \Delta a_{e}=a_{e}^{\exp }-a_{e}^{\mathrm{SM}}=-(8.7 \pm 3.6) \times 10^{-13}, \\
& \Delta a_{\mu}=a_{\mu}^{\exp }-a_{\mu}^{\mathrm{SM}}=(2.7 \pm 0.9) \times 10^{-9} .
\end{aligned}
$$

In our benchmarks, the contributions are too small to saturate these differences, as these were chosen to instead yield interesting $Z^{\prime}$ phenomenology. If we would aim to satisfy constraints on $(g-2)_{\mu}$, we would choose values for the chargino, neutralino, slepton and sneutrino masses consistent with anomalous magnetic moment constraints. Since the chargino and neutralino sector masses are not directly connected to the $Z^{\prime}$ boson mass, they would affect the $Z^{\prime}$ phenomenology through decays. As the chargino-sneutrino loop is expected to be similar to the one in MSSM, the difference will arise from the lightest neutralino contribution. Its mass and composition are important, and so is the mass of the slepton. In addition, in both MSSM and $U(1)^{\prime}$ models, the anomalous magnetic moment depends almost linearly on $\tan \beta[99,100]$. A larger value of $\tan \beta$ may increase the contributions to $(g-2)_{\mu}$, but for our benchmarks, this is in conflict with flavor constraints.

Both ATLAS and CMS collaborations have searched for $Z^{\prime}$ bosons. The assumption is that they are produced in $p p$, then decay into SM particles. The decay channels explored are $j j$ [101-103], $b \bar{b}$ [104], $t \bar{t}[105,106], e^{+} e^{-}[54,107]$, $\mu^{+} \mu^{-}$[54,107], $\tau^{+} \tau^{-}[57,108], W^{+} W^{-}[109,110]$, and $Z H_{1}^{0}$ [110], within a variety of models with extended $U(1)^{\prime}$ and $S U(2)$ gauge groups. Of these channels, the leptonic decays $e^{+} e^{-}$and $\mu^{+} \mu^{-}$impose the most stringent constraints on the $Z^{\prime}$ mass, normally $M_{Z^{\prime}} \gtrsim 4.3 \mathrm{TeV}$. However, all these analyses assumed non-supersymmetric scenarios. It has been shown that, including supersymmetry, these bounds can be reduced by $\sim 300 \mathrm{GeV}$ [63]. Hence, as we wish to explore the largest parameter space possible, we shall assume that $M_{Z^{\prime}} \geq 4.0 \mathrm{TeV}$.

We calculated the branching ratios of the $Z^{\prime}$ decaying into various final states for the three selected benchmark points, and show the results for the dominant ones in Table 5. As expected, branching ratios for decays into quarks (BP2 and BP3) or neutrinos (BP1) dominate over those into supersymmetric particles. The benchmarks were chosen for nonnegligible decays into SUSY particles pairs, and are dominated by decays into sneutrinos and chargino pairs (BP1), chargino and neutralino pairs (BP2), and into slepton pairs (BP3). In Table 5 we also test the width/mass ratio. For all benchmarks considered, $\Gamma_{Z^{\prime}} / M_{Z^{\prime}}$ remains safely under $10 \%$, justifying treating $Z^{\prime}$ as a narrow resonance. The branching ratios of $Z^{\prime}$ are very sensitive to variations in $Q_{Q}^{\prime}$, the $U(1)^{\prime}$ charge for the left-handed quark doublet. To highlight $Z^{\prime}$ decays into supersymmetric channels, we fixed all parameters for the chosen benchmarks BP1, BP2 and BP3 as in Table 2, except $Q_{Q}^{\prime}$, which is allowed to vary freely. In Fig. 1 we plot the branching ratios of the $Z^{\prime}$ as a function of $Q_{Q}^{\prime}$ for each benchmark scenario. The particular choice for $Q_{Q}^{\prime}$ for each benchmark as given in Table 2 , is obtained by requiring that some branching ratios into supersymmetric particles be maximal. We indicated these choices in each panel of Fig. 1 as a vertical grey line.

Typically, the SUSY decay modes include (i) $Z^{\prime} \rightarrow$ $\tilde{v}_{\ell_{R}} \tilde{v}_{\ell_{R}} \rightarrow 2 \ell+E_{T}$ or $4 \ell+E_{T}$, (ii) $Z^{\prime} \rightarrow \tilde{\chi}_{1}^{ \pm} \tilde{\chi}_{1}^{\mp} \rightarrow$ $2 \ell+E_{T}$, (iii) $Z^{\prime} \rightarrow \tilde{\chi}_{2}^{0} \tilde{\chi}_{3}^{0} \rightarrow 2 \ell+E_{T}$, (iv) $Z^{\prime} \rightarrow \tilde{\ell}_{R} \tilde{\ell}_{R} \rightarrow$ $2 \ell+E_{T}$, or $4 \ell+E_{T}$, or $6 \ell+E_{T}$ etc. Such pure leptonic modes give rise to a signature consisting of charged-leptons and large missing energies, which are particularly well suited for observation at the LHC. To determine and classify all possible signals for the three scenarios we look into the decay 
Table 5 Decay width (in $\mathrm{GeV}$ ), width over mass ratios, and dominant branching ratios (in \%) of $Z^{\prime}$ boson decay channels for the three scenarios considered. The total branching ratios for decay modes with $\mathrm{BR}_{i}<1 \%$ are also shown separately

\begin{tabular}{|c|c|}
\hline Width $[\mathrm{GeV}]$ and branching ratios $[\%]$ & BP1 \\
\hline$\Gamma_{Z^{\prime}}$ & 386 \\
\hline$\Gamma_{Z^{\prime}} / M_{Z^{\prime}}[\%]$ & 9.0 \\
\hline $\operatorname{BR}\left(Z^{\prime} \rightarrow \sum_{\ell} \tilde{v}_{\ell_{R}} \tilde{v}_{\ell_{R}}\right)$ & 15.69 \\
\hline $\operatorname{BR}\left(Z^{\prime} \rightarrow \tilde{\chi}_{1}^{ \pm} \tilde{\chi}_{1}^{\mp}\right)$ & 2.93 \\
\hline $\operatorname{BR}\left(Z^{\prime} \rightarrow \tilde{\chi}_{3}^{0} \tilde{\chi}_{4}^{0}\right)$ & 2.09 \\
\hline $\operatorname{BR}\left(Z^{\prime} \rightarrow \sum_{\ell} v_{\ell} \bar{v}_{\ell}\right)$ & 38.70 \\
\hline $\mathrm{BR}\left(Z^{\prime} \rightarrow \sum_{q} q_{d} \bar{q}_{d}\right)$ & 15.39 \\
\hline $\mathrm{BR}\left(Z^{\prime} \rightarrow \sum_{q} q_{u} \bar{q}_{u}\right)$ & 12.33 \\
\hline $\mathrm{BR}\left(Z^{\prime} \rightarrow \sum_{\ell} \ell \bar{\ell}\right)$ & 4.08 \\
\hline$\sum_{i}\left[\mathrm{BR}_{\mathrm{i}}\left(Z^{\prime} \rightarrow\right.\right.$ others $\left.)<1 \%\right]$ & 8.79 \\
\hline Width $[\mathrm{GeV}]$ and branching ratios $[\%]$ & $\mathrm{BP} 2$ \\
\hline$\Gamma_{Z^{\prime}}$ & 70.8 \\
\hline$\Gamma_{Z^{\prime}} / M_{Z^{\prime}}[\%]$ & 1.7 \\
\hline $\operatorname{BR}\left(Z^{\prime} \rightarrow \tilde{\chi}_{1}^{ \pm} \tilde{\chi}_{1}^{\mp}\right)$ & 5.27 \\
\hline $\mathrm{BR}\left(Z^{\prime} \rightarrow \tilde{\chi}_{2}^{0} \tilde{\chi}_{3}^{0}\right)$ & 4.09 \\
\hline $\mathrm{BR}\left(Z^{\prime} \rightarrow H^{+} H^{-}\right)$ & 1.00 \\
\hline $\mathrm{BR}\left(Z^{\prime} \rightarrow H_{2}^{0} A_{1}^{0}\right)$ & 1.26 \\
\hline $\mathrm{BR}\left(Z^{\prime} \rightarrow \sum_{q} q_{u} \bar{q}_{u}\right)$ & 36.60 \\
\hline $\mathrm{BR}\left(Z^{\prime} \rightarrow \sum_{\ell} \nu_{\ell} \bar{v}_{\ell}\right)$ & 28.98 \\
\hline $\mathrm{BR}\left(Z^{\prime} \rightarrow \sum_{q} q_{d} \bar{q}_{d}\right)$ & 12.24 \\
\hline $\mathrm{BR}\left(Z^{\prime} \rightarrow W^{+} W^{-}\right)$ & 1.42 \\
\hline $\mathrm{BR}\left(Z^{\prime} \rightarrow Z H_{1}^{0}\right)$ & 1.21 \\
\hline $\mathrm{BR}\left(Z^{\prime} \rightarrow \sum_{\ell} \ell \bar{\ell}\right)$ & 3.72 \\
\hline$\sum_{i}\left[\mathrm{BR}_{\mathrm{i}}\left(Z^{\prime} \rightarrow\right.\right.$ others $\left.)<1 \%\right]$ & 4.21 \\
\hline Width $[\mathrm{GeV}]$ and branching ratios $[\%]$ & $\mathrm{BP} 3$ \\
\hline$\Gamma_{Z^{\prime}}$ & 351 \\
\hline$\Gamma_{Z^{\prime}} / M_{Z^{\prime}}[\%]$ & 6.7 \\
\hline $\operatorname{BR}\left(Z^{\prime} \rightarrow \sum_{\ell=1}^{2} \tilde{\ell}_{R} \tilde{\ell}_{R}\right)$ & 6.02 \\
\hline $\mathrm{BR}\left(Z^{\prime} \rightarrow \tilde{\tau}_{1} \tilde{\tau}_{1}\right)$ & 3.01 \\
\hline $\mathrm{BR}\left(Z^{\prime} \rightarrow \tilde{\chi}_{2}^{0} \tilde{\chi}_{4}^{0}\right)$ & 1.02 \\
\hline $\operatorname{BR}\left(Z^{\prime} \rightarrow \sum_{\ell=1}^{2} \tilde{\ell}_{L} \tilde{\ell}_{L}\right)$ & 2.02 \\
\hline $\operatorname{BR}\left(Z^{\prime} \rightarrow \tilde{\tau}_{2} \tilde{\tau}_{2}\right)$ & 1.01 \\
\hline $\operatorname{BR}\left(Z^{\prime} \rightarrow \sum_{\ell} \tilde{v}_{\ell_{L}} \tilde{v}_{\ell_{L}}\right)$ & 3.03 \\
\hline $\mathrm{BR}\left(Z^{\prime} \rightarrow \sum_{q} q_{u} \bar{q}_{u}\right.$ & 34.50 \\
\hline $\mathrm{BR}\left(Z^{\prime} \rightarrow \sum_{\ell} \ell \bar{\ell}\right)$ & 29.22 \\
\hline $\operatorname{BR}\left(Z^{\prime} \rightarrow \sum_{\ell} \nu_{\ell} \bar{\nu}_{\ell}\right)$ & 10.29 \\
\hline$\sum_{i}\left[\mathrm{BR}_{\mathrm{i}}\left(Z^{\prime} \rightarrow\right.\right.$ others $\left.)<1 \%\right]$ & 9.88 \\
\hline
\end{tabular}

topology of these particles. We classify signals according to the final number of leptons present in the signal events. The generic Feynman diagrams contributing dominantly to chan- nels leading to signals with leptons and missing energy are shown in Fig. 2 for BP1 (a), BP2 (b) and BP3 (c).

The events are generated at the partonic level with Cal CHEP [77] and they are subsequently passed to PYTHIA 8 $[79,80]$ for decay, showering and hadronization. Events which are saved in HepMC format [111] are then passed to MadAnalysis [84] for applying cuts and further data analysis. Delphes [81-83] is used for fast detector simulations. We simulated events for the $2 \ell+E_{T}, 4 \ell+E_{T}$ and $6 \ell+E_{T}$ signals at the LHC with $14 \mathrm{TeV}$ center-of-mass energy, the HE-LHC with $27 \mathrm{TeV}$, and as well as the FCC-hh with $100 \mathrm{TeV}$. We used different PDF sets to model parton distributions for the colliders at different center of mass energies. While CTEQ611 PDF set [112] was used for the $14 \mathrm{TeV}$ LHC case, the PDF set from the PDF4LHC15 collaboration [113] was used for both HE-LHC and FCC-hh. In the numerical study, for the calculation of signal significance, we have taken the integrated luminosities $\mathcal{L}=3 \mathrm{ab}^{-1}, 15$ $\mathrm{ab}^{-1}$, and $30 \mathrm{ab}^{-1}$ for the HL-LHC, HE-LHC, and FCC-hh, respectively.

For each benchmark, we use two different formulas for the significance of the signals [114-116], where the first expression corresponds to the usual definition, and the second is more useful for smaller number of background events:

$\sigma_{\mathcal{A}}=\frac{S}{\sqrt{S+B}}$

$\sigma_{\mathcal{B}}=\sqrt{2(S+B) \log \left[\frac{(S+B)\left(B+\sigma_{B}^{2}\right)}{B^{2}+(S+B) \sigma_{B}^{2}}\right]-\frac{2 B^{2}}{\sigma_{B}^{2}} \log \left[1+\frac{S \sigma_{B}^{2}}{B\left(B+\sigma_{B}^{2}\right)}\right]}$,

where $S$ is the number of signal events, $B$ the number of background events, and $\sigma_{B}^{2}$ the standard deviation for background events. We generated the events for the signal in each scenario, and we also simulated the SM background for the three benchmarks, separately for 14, 27 and $100 \mathrm{TeV}$.

We concentrate on leptonic final states, considered as golden channels in experimental searches at LHC. To exploit these features, this study will be focused on the decays of the $Z^{\prime}$ boson into supersymmetric particles, leading to final states with leptons and missing energy, due to the presence of neutralinos or neutrinos. In the following, we present a study of $Z^{\prime}$ decays into multileptonic final states for a given set of the secluded sector $U(1)^{\prime}$ model parameters (BP1, BP2 and BP3), dividing our analysis into $2 \ell+\mathbb{E}_{T}, 4 \ell+\mathbb{E}_{T}$ and $6 \ell+E_{T}$ signals.

\subsection{Multilepton analysis}

In this analysis, for each final state, we impose cuts on the kinematical observables to suppress the SM background, as given in Table 6. Given the event topologies, stricter cuts on the leading lepton transverse momentum favor events with 

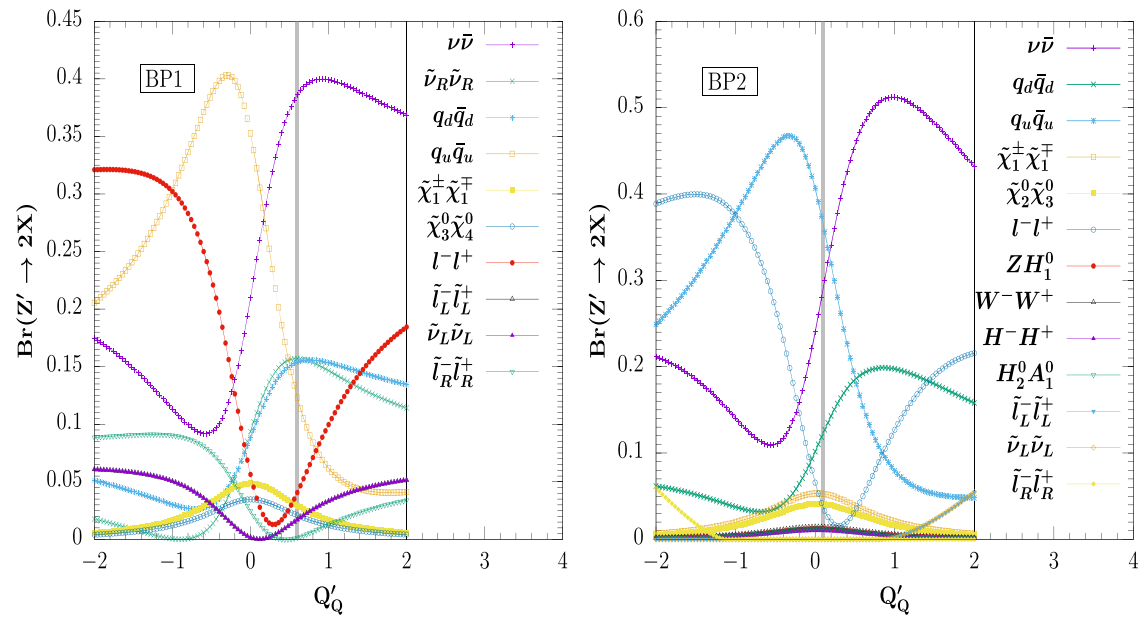

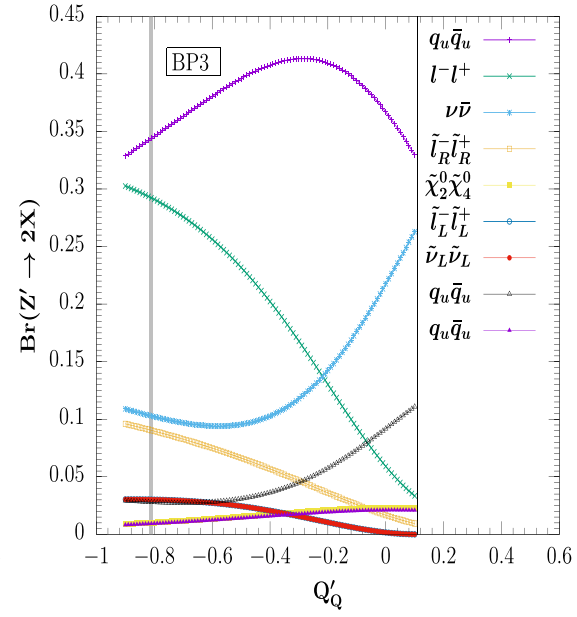

Fig. $1 Z^{\prime}$ decay branching ratios as a function of $Q_{Q}^{\prime}$ for BP1 (left), BP2 (middle) and BP3 (right). For each benchmark we fix all other parameters as in Table 2 except for $Q_{Q}^{\prime}$, which is allowed to vary. The choice of $Q_{Q}^{\prime}$ for each benchmark, chosen to maximize decays into supersymmetric particles, is indicated in each panel as a vertical grey line. Note that the branching ratios of $Z^{\prime}$ into the SM fermions and their superpartners as well as into the right-handed scalar neutrinos are all depicted after summing over three generations

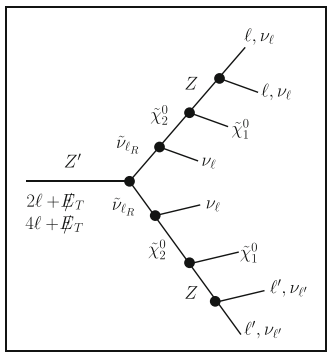

(a)

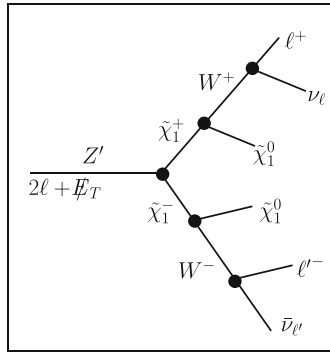

(b)

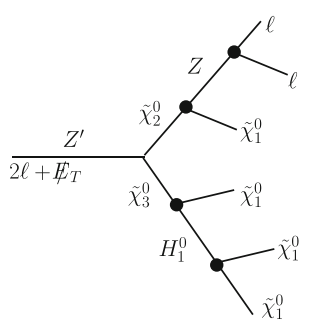

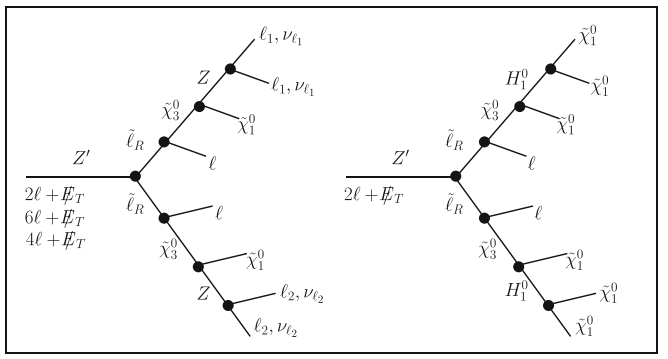

(c)

Fig. 2 The generic Feynman diagrams for the decay channels of the $Z^{\prime}$ in the secluded $U(1)^{\prime}$ model for BP1, BP2, and BP3 in a-c, respectively

$2 \ell+E_{T}$ and $4 \ell+E_{T}$. While the cuts on the angular variables and lepton separation remain the same, the kinematic cuts increase (in general), as expected going from 14 to $27 \mathrm{TeV}$ and eventually to $100 \mathrm{TeV}$. Very stringent cuts are needed for the case of $2 \ell+E_{T}$ signal, and this is valid for the three center of mass energies but especially at $100 \mathrm{TeV}$. The final set of cuts are obtained by requiring to maximize the signal significance. We proceed in turn to analyze each of the final states, $2 \ell+\mathbb{E}_{T}, 4 \ell+\mathbb{E}_{T}$ and $6 \ell+\mathbb{E}_{T}$ signals and discuss their potential for observability.

\subsubsection{Two lepton signal: $2 \ell+\not E_{T}$}

The main decay modes of $Z^{\prime}$ giving rise to dilepton final states are:

$$
\begin{aligned}
& Z^{\prime} \rightarrow \tilde{v}_{\ell_{R}} \tilde{v}_{\ell_{R}} \rightarrow 2 \ell+E_{T}, \\
& Z^{\prime} \rightarrow \tilde{\chi}_{1}^{ \pm} \tilde{\chi}_{1}^{\mp} \rightarrow 2 \ell+E_{T}, \\
& Z^{\prime} \rightarrow \tilde{\chi}_{2}^{0} \tilde{\chi}_{3}^{0} \rightarrow 2 \ell+E_{T}, \\
& Z^{\prime} \rightarrow \tilde{\ell}_{R} \tilde{\ell}_{R} \rightarrow 2 \ell+E_{T} .
\end{aligned}
$$

In the following figures, we first show the relevant kinematic variables for signals and background at 14, 27 and $100 \mathrm{TeV}$, before any cuts were imposed. We plot the differential cross-section, normalized to unity, with individual bin contents divided by the sum of all the data in the available bins. This way, the uncertainties are correlated across the bins, such that the uncertainties on the total integrated luminosity cancel. The resulting normalized differential fiducial cross-section is plotted as a function of various representative kinematic variables $[117,118]$. Let us define the variable transverse mass $M_{T}$ [119]

$$
M_{T}(\ell)=\sqrt{\left(E_{T}(\ell)+\mathbb{E}_{T}\right)^{2}-\left(\mathbf{p}_{T}(\ell)+\mathbf{E}_{T}\right)^{2}}
$$

for a system composed of a lepton $\ell$ and the invisible transverse momentum available in each event. Here $\mathbb{E}_{T}=\left|\mathbf{E}_{T}\right|$. We show, in Fig. 3, $M_{T}$ of the leading lepton (top panels) and next-to-leading lepton (bottom panels), and in Fig. 4, the leading lepton transverse momentum (top panels) and next-to-leading lepton transverse momentum (bottom pan- 
Table 6 The set of kinematical cuts used to isolate signal events from background

\begin{tabular}{|c|c|c|}
\hline @ $14 \mathrm{TeV}$ & @27 TeV & @ $100 \mathrm{TeV}$ \\
\hline \multicolumn{3}{|l|}{$2 \ell+E_{T}$} \\
\hline$|\eta|<2.5$ & $|\eta|<2.5$ & $|\eta|<2.5$ \\
\hline$\Delta R_{\ell \ell} \geq 0.5$ & $\Delta R_{\ell \ell} \geq 0.5$ & $\Delta R_{\ell \ell} \geq 0.5$ \\
\hline$p_{T}\left(\ell_{1}\right)>475 \mathrm{GeV}$ & $p_{T}\left(\ell_{1}\right)>500 \mathrm{GeV}$ & $p_{T}\left(\ell_{1}\right)>2000 \mathrm{GeV}$ \\
\hline$p_{T}\left(\ell_{2}\right)>50 \mathrm{GeV}$ & $p_{T}\left(\ell_{2}\right)>300 \mathrm{GeV}$ & $p_{T}\left(\ell_{2}\right)>1000 \mathrm{GeV}$ \\
\hline$E_{T}>50 \mathrm{GeV}$ & $E_{T}>400 \mathrm{GeV}$ & $\not_{T}>2300 \mathrm{GeV}$ \\
\hline \multicolumn{3}{|l|}{$4 \ell+E_{T}$} \\
\hline$|\eta|<2.5$ & $|\eta|<2.5$ & $|\eta|<2.5$ \\
\hline$\Delta R_{\ell \ell} \geq 0.5$ & $\Delta R_{\ell \ell} \geq 0.5$ & $\Delta R_{\ell \ell} \geq 0.5$ \\
\hline$p_{T}\left(\ell_{1}\right)>100 \mathrm{GeV}$ & $p_{T}\left(\ell_{1}\right)>100 \mathrm{GeV}$ & $p_{T}\left(\ell_{1}\right)>100 \mathrm{GeV}$ \\
\hline$p_{T}\left(\ell_{2}\right)>50 \mathrm{GeV}$ & $p_{T}\left(\ell_{2}\right)>50 \mathrm{GeV}$ & $p_{T}\left(\ell_{2}\right)>50 \mathrm{GeV}$ \\
\hline$p_{T}\left(\ell_{3}\right)>25 \mathrm{GeV}$ & $p_{T}\left(\ell_{3}\right)>25 \mathrm{GeV}$ & $p_{T}\left(\ell_{3}\right)>25 \mathrm{GeV}$ \\
\hline$p_{T}\left(\ell_{4}\right)>15 \mathrm{GeV}$ & $p_{T}\left(\ell_{4}\right)>15 \mathrm{GeV}$ & $p_{T}\left(\ell_{4}\right)>15 \mathrm{GeV}$ \\
\hline$E_{T}>400 \mathrm{GeV}$ & $E_{T}>350 \mathrm{GeV}$ & $\mathbb{E}_{T}>800 \mathrm{GeV}$ \\
\hline \multicolumn{3}{|l|}{$6 \ell+E_{T}$} \\
\hline$|\eta|<2.5$ & $|\eta|<2.5$ & $|\eta|<2.5$ \\
\hline$\Delta R_{\ell \ell} \geq 0.2$ & $\Delta R_{\ell \ell} \geq 0.2$ & $\Delta R_{\ell \ell} \geq 0.2$ \\
\hline$p_{T}\left(\ell_{1}\right)>50 \mathrm{GeV}$ & $p_{T}\left(\ell_{1}\right)>50 \mathrm{GeV}$ & $p_{T}\left(\ell_{1}\right)>100 \mathrm{GeV}$ \\
\hline$p_{T}\left(\ell_{2}\right)>20 \mathrm{GeV}$ & $p_{T}\left(\ell_{2}\right)>20 \mathrm{GeV}$ & $p_{T}\left(\ell_{2}\right)>50 \mathrm{GeV}$ \\
\hline$p_{T}\left(\ell_{3}\right)>20 \mathrm{GeV}$ & $p_{T}\left(\ell_{3}\right)>20 \mathrm{GeV}$ & $p_{T}\left(\ell_{3}\right)>20 \mathrm{GeV}$ \\
\hline$p_{T}\left(\ell_{4}\right)>20 \mathrm{GeV}$ & $p_{T}\left(\ell_{4}\right)>20 \mathrm{GeV}$ & $p_{T}\left(\ell_{4}\right)>20 \mathrm{GeV}$ \\
\hline$p_{T}\left(\ell_{5}\right)>10 \mathrm{GeV}$ & $p_{T}\left(\ell_{5}\right)>10 \mathrm{GeV}$ & $p_{T}\left(\ell_{5}\right)>15 \mathrm{GeV}$ \\
\hline$p_{T}\left(\ell_{6}\right)>5 \mathrm{GeV}$ & $p_{T}\left(\ell_{6}\right)>5 \mathrm{GeV}$ & $p_{T}\left(\ell_{6}\right)>5 \mathrm{GeV}$ \\
\hline$E_{T}>100 \mathrm{GeV}$ & $E_{T}>100 \mathrm{GeV}$ & $E_{T}>100 \mathrm{GeV}$ \\
\hline
\end{tabular}

els). The left-hand side panels in both figures correspond to signals and backgrounds at $14 \mathrm{TeV}$, the middle at $27 \mathrm{TeV}$, and the right-hand side for $100 \mathrm{TeV}$.

The main backgrounds (di-bosons for $2 \ell+\mathbb{E}_{T}$ ) are indicated in solid lines while the signals are plotted in dotted lines, color coded: green for BP1, black for BP2 and orange for BP3. For our analysis we have checked cross sections of other potential backgrounds (tri-bosons) for $2 \ell+E_{T}$, such as $W W Z$ and $Z Z Z$ and found that their cross sections at $14 \mathrm{TeV}$ are about three orders of magnitude smaller than those of the di-bosons. Such tri-boson background suppressions hold also at $27 \mathrm{TeV}$ and $100 \mathrm{TeV}$ center of mass energies. Thus they are subdominant and ignored in our analysis. We repeated the background calculations at each energy for the other signals (namely, $4 \ell+E_{T}$ and $6 \ell+E_{T}$ ) and only the dominant backgrounds, tri-bosons for $4 \ell+E_{T}$ and $Z Z Z Z$ for $6 \ell+E_{T}$, are kept in this analysis. As our signal is leptonic only, we selected certain number of isolated leptons + MET and vetoed any jets to avoid consistently signals and backgrounds due to QCD interactions. As an extensive background calculation is beyond the scope of our work, we restricted ourselves with backgrounds without jets, thus neglecting any contribution from soft jets, or jets which will not survive cuts.

Both of these graphs show clearly that at large $M_{T}(\ell)$ and $p_{T}(\ell)$, the signal dominates the background, and the graphs justify our choice of kinematic cuts. Table 7 gives values for signal and background cross-sections after each cut. We also show the signal significance, for both $\sigma_{\mathcal{A}}$ and $\sigma_{\mathcal{B}}$, for each benchmark, at proposed total integrated luminosity: for 14 $\mathrm{TeV}$ at $\mathcal{L}=3 \mathrm{ab}^{-1}$, for $27 \mathrm{TeV}$ at $\mathcal{L}=15 \mathrm{ab}^{-1}$ and for $100 \mathrm{TeV}$ at $\mathcal{L}=30 \mathrm{ab}^{-1}$. While BP3 appears to be most promising, the significance for all benchmarks at $14 \mathrm{TeV}$ is very low, dispelling any hope for observing the $Z^{\prime}$ boson in the $2 \ell+Z_{T}$ final state. This is not surprising, and in complete agreement with other findings [63,120]. However, at $27 \mathrm{TeV}$ the cuts imposed are especially effective for BP3, and we obtain large significances for both $\sigma_{\mathcal{A}}$ and $\sigma_{\mathcal{B}}$. At $100 \mathrm{TeV}$, the cuts imposed to reduce the background wiped out the signal, and we were not able to gain any predictable features. We had here two available options: either loose all the signal events due to the stringent cuts applied, or allow the signals to be overwhelmed by large background events. 

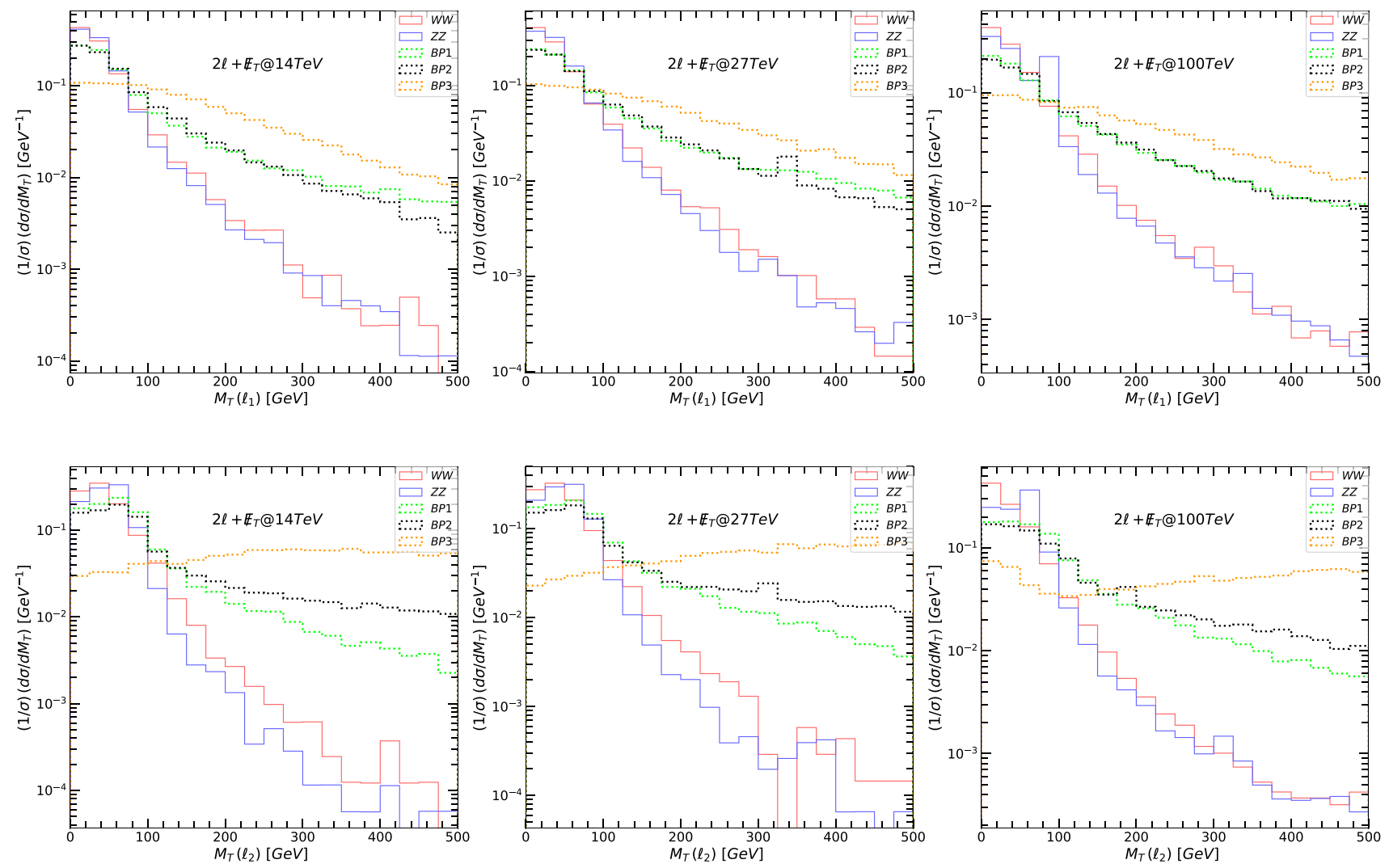

Fig. 3 The transverse mass $M_{T}$, for the leading lepton $\ell_{1}$ (top panels) and next-to-leading lepton $\ell_{2}$ (bottom panels), for the signal and background in the $2 \ell+E_{T}$ scenario. (Left-hand) signals and backgrounds at $14 \mathrm{TeV}$, (middle) at $27 \mathrm{TeV}$, and (right-hand) at $100 \mathrm{TeV}$. The main backgrounds (di-bosons) are indicated in solid lines while the signals are plotted in dotted lines: green for BP1, black for BP2 and orange for BP3

\subsubsection{Four lepton signal: $4 \ell+\mathbb{E}_{T}$}

The main decay modes of the $Z^{\prime}$ boson yielding $4 \ell+E_{T}$ signals are

$$
\begin{aligned}
& Z^{\prime} \rightarrow \tilde{v}_{\ell_{R}} \tilde{v}_{\ell_{R}} \rightarrow 4 \ell+E_{T}, \\
& Z^{\prime} \rightarrow \tilde{\ell}_{R} \tilde{\ell}_{R} \rightarrow 4 \ell+E_{T} .
\end{aligned}
$$

In the following figures, we first plot the relevant kinematic variables signals and background at 14, 27 and $100 \mathrm{TeV}$, before any cuts were imposed. We show, in Fig. 5, the total missing transverse energy $E_{T}$. For each figure we plot, in the left-hand columns, signals and backgrounds at $14 \mathrm{TeV}$, the middle columns at $27 \mathrm{TeV}$, and right-hand columns, at $100 \mathrm{TeV}$. Both these plots indicate that the signal over background, before cuts, seems most promising at higher energies, and that $E_{T}$ is overall a better variable to differentiate between signal and background. We also note that, as for the $2 \ell+E_{T}$ case, the scenario BP3 is the most promising.

In Fig. 6 we plot the transverse mass $M_{T}$ for the leading lepton $\ell_{1}$ (top row panels) and next-to-leading lepton $\ell_{2}$ (bottom row panels) for the signal and background for the

$4 \ell+E_{T}$ signal. In Fig. 7, we show the transverse momenta of the leading lepton $\ell_{1}$ (top row) and next-to-leading lepton $\ell_{2}$ (bottom), for the signal and background. The transverse momenta of the third and fourth leptons have similar distributions to the next-to leading lepton, and thus we do not plot them. The left-hand side panels in both figures correspond to signals and backgrounds at $14 \mathrm{TeV}$, the middle at $27 \mathrm{TeV}$, and the right-hand side for $100 \mathrm{TeV}$. The main backgrounds (three-bosons) are indicated in solid lines while the signals are plotted in dotted lines: green for BP1, and orange for BP3. ${ }^{4}$ These graphs show clearly that at large $M_{T}(\ell)$ and $p_{T}(\ell)$, the signal dominates the background, and the graphs justify our choice of kinematic cuts. The distribution is rather similar for the leading leptons, and the $p_{T}$ observable is better than the $M_{T}$ at distinguishing signals from the backgrounds. In all the plots, the signal from BP3 is most promising, particularly at high energy/momenta.

Table 8 gives values for signal and background cross sections after each cut. We also show the signal significance, for both $\sigma_{\mathcal{A}}$ and $\sigma_{\mathcal{B}}$, for each benchmark, at proposed total

$\overline{{ }^{4} \text { Note that } 4 \ell}+\not E_{T}$ signal is not realized in BP2 as indicated in Fig. 2. 

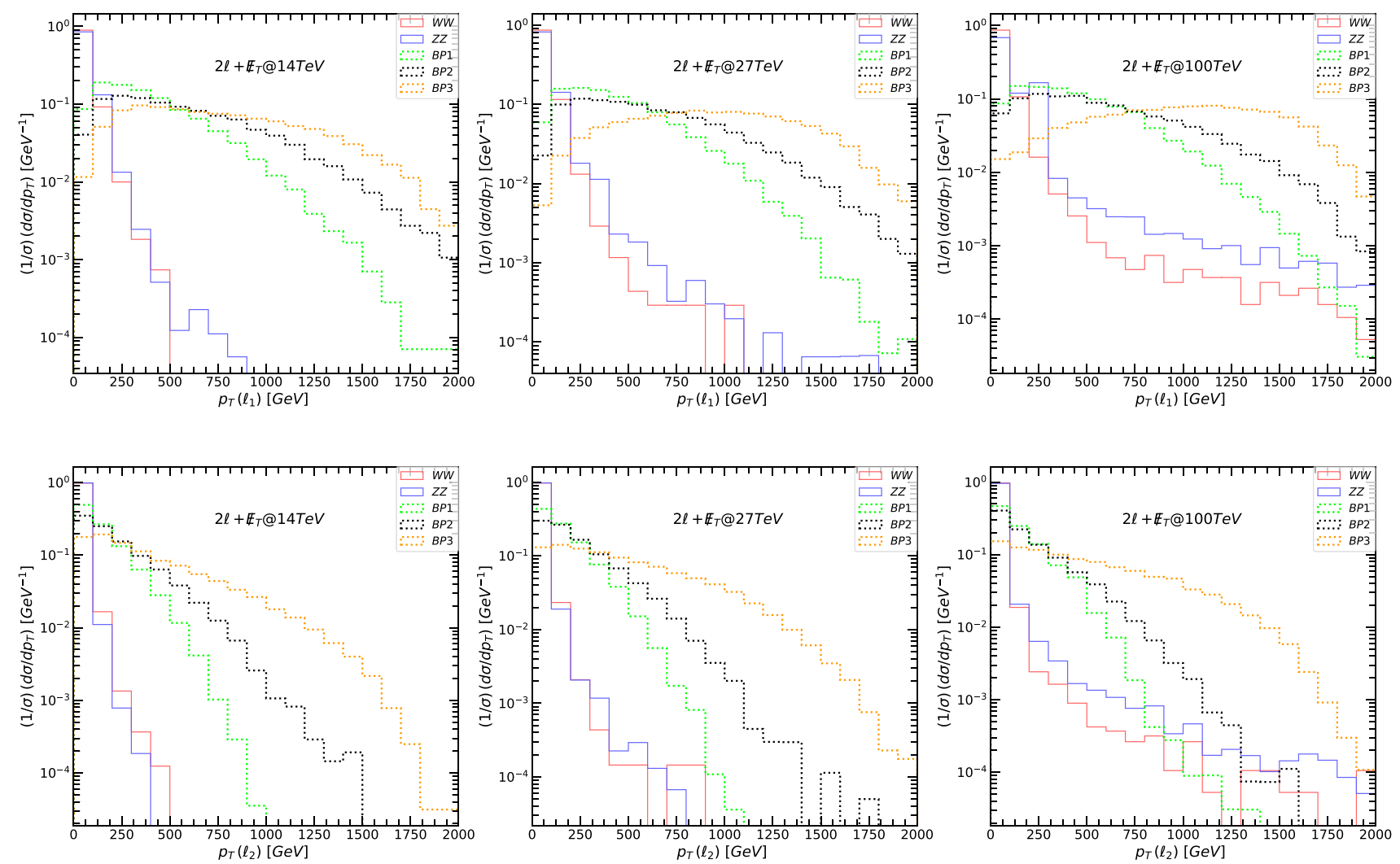

Fig. 4 The transverse momentum $p_{T}$, for the leading lepton $\ell_{1}$ (top panels) and next-to-leading lepton $\ell_{2}$ (bottom panels), for the signal and background in the $2 \ell+E_{T}$ scenario. (Left-hand) signals and backgrounds at $14 \mathrm{TeV}$, (middle) at $27 \mathrm{TeV}$, and (right-hand) at $100 \mathrm{TeV}$.
The main backgrounds (di-bosons) are indicated in solid lines while the signals are plotted in dotted lines: green for BP1, black for BP2 and orange for $\mathrm{BP} 3$ integrated luminosity: for $14 \mathrm{TeV}$ at $\mathcal{L}=3 \mathrm{ab}^{-1}$, for 27 $\mathrm{TeV}$ at $\mathcal{L}=15 \mathrm{ab}^{-1}$ and for $100 \mathrm{TeV}$ at $\mathcal{L}=30 \mathrm{ab}^{-1}$. Unlike the case of $2 \ell+E_{T}$, we keep the cuts constant for different centre-of-mass energies. Again, the significance for all benchmarks at $14 \mathrm{TeV}$ for observing the $Z^{\prime}$ boson in the $4 \ell+E_{T}$ final state is low. However, at $27 \mathrm{TeV}$ both BP1 and BP3 show some promise, and we obtain large significances of $\sim 3 \sigma$ or more for $\sigma_{\mathcal{A}}$. At $100 \mathrm{TeV}$, though there are many uncertainties and unknowns, and our results should be interpreted as estimates only, both BP1 and BP3 show significant promise for observability.

\subsubsection{Six lepton signal: $6 \ell+\mathbb{Z}_{T}$}

The dominant decay mode of the $Z^{\prime}$ gauge boson, yielding a $6 \ell+\not \not_{T}$ signal is

$Z^{\prime} \rightarrow \tilde{\ell}_{R} \tilde{\ell}_{R} \rightarrow 6 \ell+E_{T}$.

Quite clearly, the $6 \ell+\mathbb{E}_{T}$ signal requires that this decay have a non-negligible branching ratio, which occurs for the BP3 scenario, as seen from Table 5, where $Z^{\prime} \rightarrow \tilde{\ell}_{R} \tilde{\ell}_{R} \sim 3 \%$. Thus as expected, this will be the only signal of interest for the $6 \ell+\mathbb{E}_{T}$ signal. In Fig. 8 we plot the missing transverse energy $E_{T}$, for the signal and background for the $6 \ell+E_{T}$ signal: (left-hand) signals and backgrounds at $14 \mathrm{TeV}$, (middle) at $27 \mathrm{TeV}$, and (right-hand) at $100 \mathrm{TeV}$. We show the main (four-bosons) backgrounds in solid lines while the the signal BP3 is given by a dotted green line.

In Fig. 9 we plot transverse momentum $p_{T}$ for the leading lepton $\ell_{1}$ (top panels), the next-to-leading lepton $\ell_{2}$ (second panels), and the next-to-next to leading lepton $\ell_{3}$ (bottom panels) for the signal and background for the $6 \ell+E_{T}$ signal. The transverse momentum $p_{T}$ plots for the fourth, fifth, and sixth lepton are similar and we do not show them here. As before the left side panels indicate signals and backgrounds at $14 \mathrm{TeV}$, the middle at $27 \mathrm{TeV}$, and the right side at $100 \mathrm{TeV}$. The main backgrounds (four-bosons) are indicated in solid lines while the dotted green line represent the signal BP3. As expected, the leading lepton $p_{T}$ distribution is most promising in distinguishing this signal from background, with other lepton $p_{T}$ distributions slightly less so.

Similar to the $2 \ell+\not_{T}$ and $4 \ell+\not_{T}$ signals, the effects of various cuts on cross sections are listed in Table 9. We also show the signal significance, for both $\sigma_{\mathcal{A}}$ and $\sigma_{\mathcal{B}}$, for BP3, 
Table 7 Signal selection strategy and cuts imposed in the $2 \ell+E_{T}$ scenario at 14,27 and $100 \mathrm{TeV}$. We give the cross-section for background and benchmark scenarios in fb. Statistical significances $\sigma_{\mathcal{A}}$ and $\sigma_{\mathcal{B}}$ of $2 \ell+\mathbb{E}_{T}$ signal are given for each energy

\begin{tabular}{|c|c|c|c|c|}
\hline $2 \ell+E_{T} @ 14 \mathrm{TeV}$ & Background [fb] & $\mathrm{BP} 1[\mathrm{fb}]$ & $\mathrm{BP} 2[\mathrm{fb}]$ & $\mathrm{BP} 3[\mathrm{fb}]$ \\
\hline No cut & $7.15 \times 10^{2}$ & $2.26 \times 10^{-2}$ & $8.25 \times 10^{-3}$ & $9.11 \times 10^{-3}$ \\
\hline$\left|\eta_{i}\right|<2.5, \Delta R_{12} \geq 0.5$ & $6.99 \times 10^{2}$ & $1.14 \times 10^{-2}$ & $4.54 \times 10^{-3}$ & $9.06 \times 10^{-3}$ \\
\hline$p_{T}\left(\ell_{1}\right)>475 \mathrm{GeV}$ & $7.13 \times 10^{-2}$ & $1.00 \times 10^{-3}$ & $1.61 \times 10^{-3}$ & $6.29 \times 10^{-3}$ \\
\hline$p_{T}\left(\ell_{2}\right)>50 \mathrm{GeV}$ & $7.13 \times 10^{-2}$ & $1.52 \times 10^{-4}$ & $1.28 \times 10^{-3}$ & $6.01 \times 10^{-3}$ \\
\hline$E_{T}>50 \mathrm{GeV}$ & $7.10 \times 10^{-2}$ & $1.5 \times 10^{-4}$ & $1.3 \times 10^{-3}$ & $5.9 \times 10^{-3}$ \\
\hline Significance: $\sigma_{\mathcal{A}}$ & $\mathcal{L}=3 \mathrm{ab}^{-1}$ & $0.031 \sigma$ & $0.26 \sigma$ & $1.2 \sigma$ \\
\hline$\sigma_{\mathcal{B}}$ & & $0.022 \sigma$ & $0.18 \sigma$ & $0.85 \sigma$ \\
\hline \multicolumn{5}{|l|}{$2 \ell+E_{T} @ 27 \mathrm{TeV}$} \\
\hline No cut & $1.59 \times 10^{3}$ & $3.54 \times 10^{-1}$ & $1.25 \times 10^{-1}$ & $1.94 \times 10^{-1}$ \\
\hline$\left|\eta_{i}\right|<2.5, \Delta R_{12} \geq 0.5$ & $1.55 \times 10^{3}$ & $1.52 \times 10^{-1}$ & $6.43 \times 10^{-2}$ & $1.94 \times 10^{-1}$ \\
\hline$p_{T}\left(\ell_{1}\right)>500 \mathrm{GeV}$ & 2.14 & $1.54 \times 10^{-2}$ & $1.18 \times 10^{-2}$ & $1.6 \times 10^{-1}$ \\
\hline$p_{T}\left(\ell_{2}\right)>300 \mathrm{GeV}$ & $9.22 \times 10^{-1}$ & $3.35 \times 10^{-4}$ & $9.76 \times 10^{-3}$ & $1.12 \times 10^{-1}$ \\
\hline$\not_{T}>400 \mathrm{GeV}$ & $1.8 \times 10^{-1}$ & $1.0 \times 10^{-4}$ & $4.4 \times 10^{-3}$ & $6.0 \times 10^{-2}$ \\
\hline Significance: $\sigma_{\mathcal{A}}$ & $\mathcal{L}=15 \mathrm{ab}^{-1}$ & $0.029 \sigma$ & $1.2 \sigma$ & $14.9 \sigma$ \\
\hline$\sigma_{\mathcal{B}}$ & & $0.021 \sigma$ & $0.88 \sigma$ & $11.3 \sigma$ \\
\hline \multicolumn{5}{|l|}{$2 \ell+\not E_{T} @ 100 \mathrm{TeV}$} \\
\hline No cut & $1.89 \times 10^{4}$ & 9.11 & 3.23 & 6.52 \\
\hline$\left|\eta_{i}\right|<2.5, \Delta R_{12} \geq 0.5$ & $8.41 \times 10^{3}$ & 3.36 & $6.62 \times 10^{-1}$ & 5.75 \\
\hline$p_{T}\left(\ell_{1}\right)>2000 \mathrm{GeV}$ & $2.01 \times 10^{1}$ & 0.0 & $1.62 \times 10^{-3}$ & $5.15 \times 10^{-2}$ \\
\hline$p_{T}\left(\ell_{2}\right)>1000 \mathrm{GeV}$ & 8.94 & 0.0 & $1.2 \times 10^{-4}$ & $1.5 \times 10^{-2}$ \\
\hline$\not_{T}>2300 \mathrm{GeV}$ & $8.1 \times 10^{-1}$ & 0.0 & 0.0 & $3.5 \times 10^{-4}$ \\
\hline Significance: $\sigma_{\mathcal{A}}$ & $\mathcal{L}=30 \mathrm{ab}^{-1}$ & $0.0 \sigma$ & $0.0 \sigma$ & $0.067 \sigma$ \\
\hline$\sigma_{\mathcal{B}}$ & & $0.0 \sigma$ & $0.0 \sigma$ & $0.048 \sigma$ \\
\hline
\end{tabular}
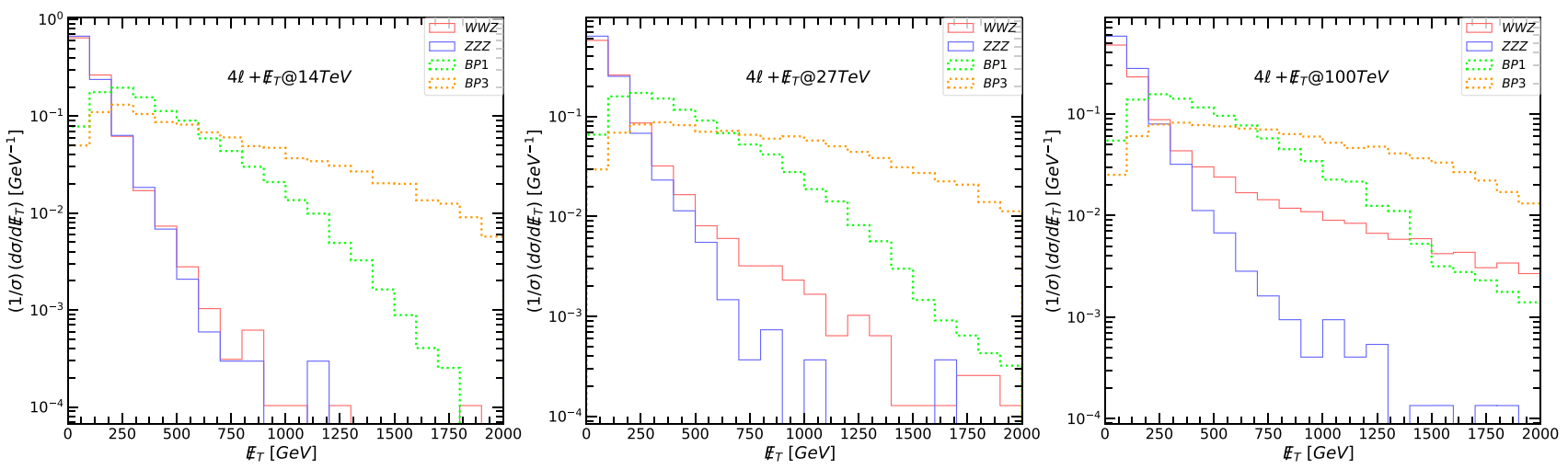

Fig. 5 The total missing energy $E_{T}$ for the signal and background for the $4 \ell+Z_{T}$ signal. (Left-hand) signals and backgrounds at $14 \mathrm{TeV}$, (three-bosons) are indicated in solid lines while the signals are plotted (middle) at $27 \mathrm{TeV}$, and (right-hand) at $100 \mathrm{TeV}$. The main backgrounds 

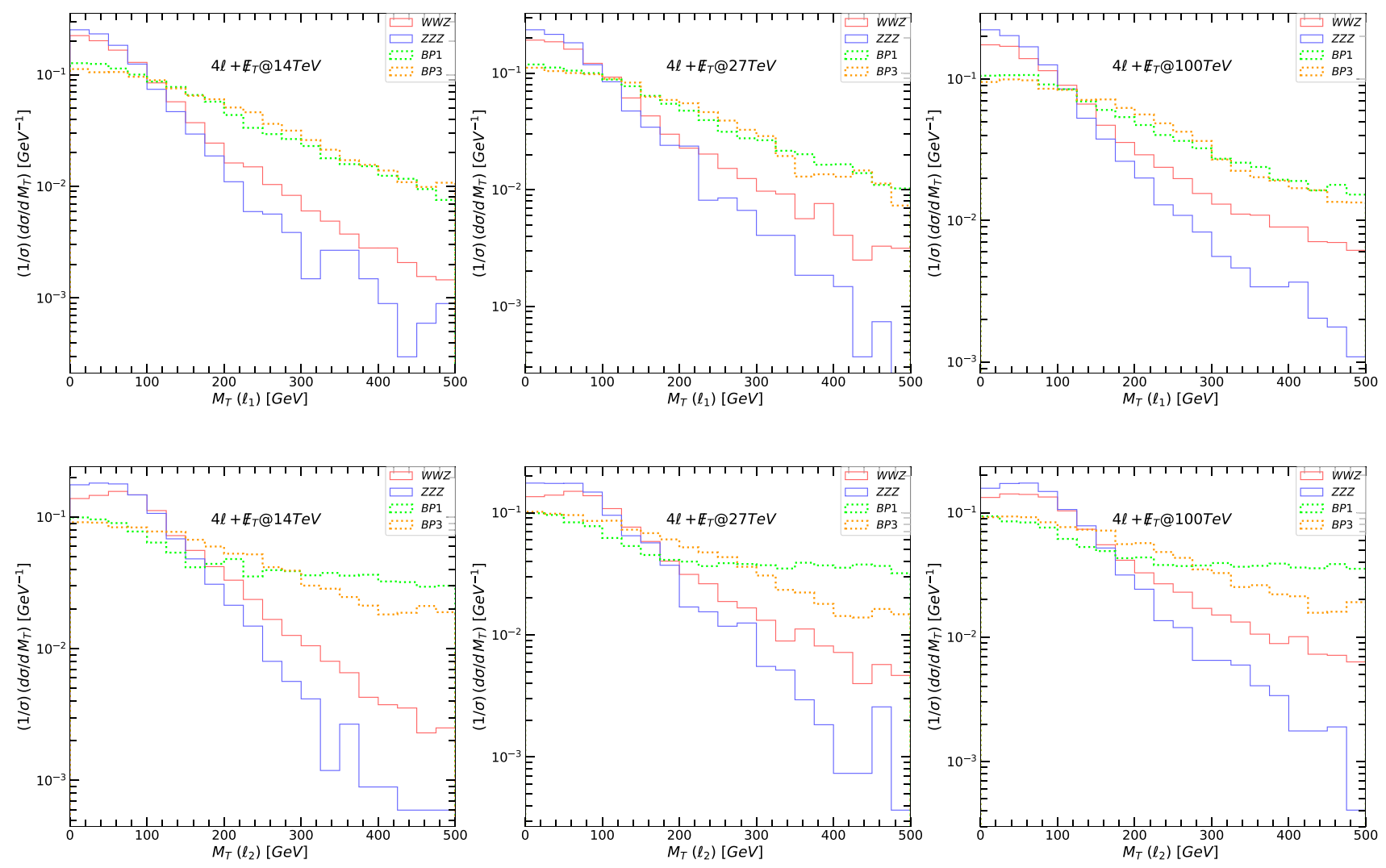

Fig. 6 The transverse mass $M_{T}$, for the leading lepton $\ell_{1}$ (top row) and next-to-leading lepton $\ell_{2}$ (bottom row), for the signal and background for the $4 \ell+E_{T}$ signal. (Left-hand) signals and backgrounds at $14 \mathrm{TeV}$, (middle) at $27 \mathrm{TeV}$, and (right-hand) at $100 \mathrm{TeV}$. The main backgrounds (three-bosons) are indicated in solid lines while the signals are plotted in dotted lines: green for BP1, and orange for BP3 at proposed total integrated luminosity: for $14 \mathrm{TeV}$ at $\mathcal{L}=3$ $\mathrm{ab}^{-1}$, for $27 \mathrm{TeV}$ at $\mathcal{L}=15 \mathrm{ab}^{-1}$ and for $100 \mathrm{TeV}$ at $\mathcal{L}=30$ $\mathrm{ab}^{-1}$. The signal significance can be around $3 \sigma$ at $27 \mathrm{TeV}$ and even greater than $8 \sigma$ at $100 \mathrm{TeV}$.

\section{Summary and conclusion}

In this work we have analyzed the LHC, HE-LHC and FCChh discovery prospects of a new neutral gauge boson $\left(Z^{\prime}\right)$ through its supersymmetric decay modes, as a promising signal for supersymmetry in an extended gauge structure. We have assumed that the $Z^{\prime}$ originates from an additional $U(1)^{\prime}$ symmetry, and we decoupled its mass scale from that of supersymmetry breaking, assumed to be the same as the scale of breaking $U(1)^{\prime}$. This allows the $Z^{\prime}$ boson to be heavy, as indicated by lower limits $M_{Z^{\prime}} \gtrsim 4 \mathrm{TeV}$ from the experimental searches, while the electroweakinos remain light. For this, we relied on the secluded $U(1)^{\prime}$ model, where three additional singlet superfields are added to the model. Unlike the VEV of the singlet scalar field which breaks $U(1)^{\prime}$ and affects the mass of supersymmetric particles, the VEVs of the additional scalars enter only in the expression for the $Z^{\prime}$ mass.

This scenario provides a fertile ground for analyzing $Z^{\prime}$ decays into chargino, neutralino, slepton and sneutrino pairs. As LHC is particularly sensitive to events containing one or more leptons, we looked for production of $Z^{\prime}$ followed by decays into multileptons plus missing energy. For this, we devised three benchmarks (BP1, BP2 and BP3) where branching ratios into some supersymmetric particles are enhanced. For instance in BP1, the decay into sneutrinos and chargino is enhanced, in BP2 it is the decay into chargino and neutralino pairs, while for BP3, the decay into right sleptons and lightest staus is important. The benchmarks are chosen also so they satisfy collider and relic density constraints.

We proceed by analyzing the observability of the signals at $\sqrt{s}=14,27$ and $100 \mathrm{TeV}$, looking separately at $2 \ell+\mathbb{E}_{T}$, $4 \ell+E_{T}$ and $6 \ell+E_{T}$ signals. Throughout our benchmarks, the ratio $\Gamma_{Z^{\prime}} / M_{Z^{\prime}}$ remains under $10 \%$, so we can treat $Z^{\prime}$ as a narrow resonance. For each signal, we perform a Monte Carlo simulation of the signal and background, and devise cuts to disproportionately suppress the latter. We present the results before and after the cuts, and calculate the significance in two different ways. 

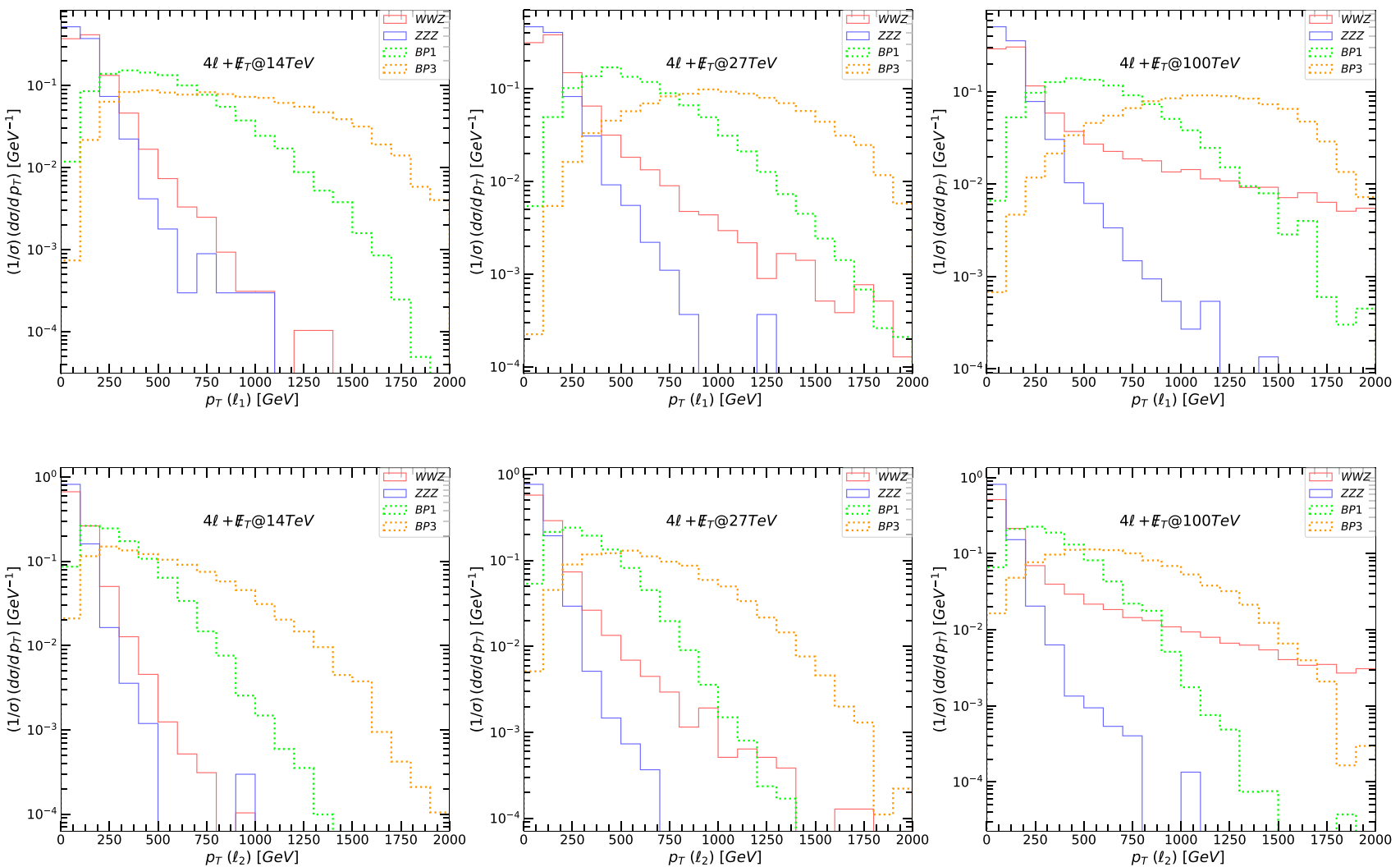

Fig. 7 The transverse momentum $p_{T}$, for the leading lepton $\ell_{1}$ (top panels) and next-to-leading lepton $\ell_{2}$ (bottom panels), for the signal and background for the $4 \ell+E_{T}$ signal. (Left-hand) signals and back- grounds at $14 \mathrm{TeV}$, (middle) at $27 \mathrm{TeV}$, and (right-hand) at $100 \mathrm{TeV}$. The main backgrounds (three-bosons) are indicated in solid lines while the signals are plotted in dotted lines: green for BP1, and orange for BP3
Overall, our findings indicate that the probability of observing $Z^{\prime}$ through supersymmetric decays at $14 \mathrm{TeV}$ is not good, even at high total integrated luminosity $\mathcal{L}=3$ $\mathrm{ab}^{-1}$. This occurs across all $2 \ell+E_{T}, 4 \ell+\not_{T}$ and $6 \ell+E_{T}$ signals and for all benchmarks. This confirms past analyses for $2 \ell+E_{T}[60,74]$, which indicated that, unless $Z^{\prime}$ is leptophobic, and thus much lighter, the signal significance is small. However, that is not necessarily so at 27 or $100 \mathrm{TeV}$ and across all signals. At $27 \mathrm{TeV}$, benchmark BP3 gives a significance well above $5 \sigma$ in $2 \ell+\mathbb{E}_{T}$ signal. While for the

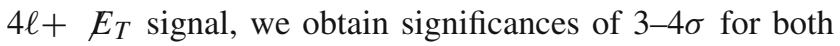
BP1 and BP3, and much higher at $100 \mathrm{TeV}$. For the $6 \ell+E_{T}$ signal, only BP3, were the $Z^{\prime}$ decay into right sleptons is important, gives any significant contributions. The signifi- cance at $27 \mathrm{TeV}$ with total integrated luminosity $\mathcal{L}=15$ $\mathrm{ab}^{-1}$ is $3-4 \sigma$, and can reach $8-9 \sigma$ at $100 \mathrm{TeV}$ with total integrated luminosity $\mathcal{L}=30 \mathrm{ab}^{-1}$.

Of course, analyses at $27 \mathrm{TeV}$ are plagued by uncertainties, and those at $100 \mathrm{TeV}$ can be interpreted as merely estimates. However, our analysis shows that HE/HL-LHC and FCChh can be promising grounds for observing consequences of both supersymmetry and extended gauge symmetry, of which an additional neutral gauge boson is one of the simplest examples. A heavy $Z^{\prime}$ boson, accompanied by a light electroweakino sector would also be indicative of a $U(1)^{\prime}$ model with a secluded sector, as this set-up facilitates the splitting of the two scales. 
Table 8 Signal selection strategy and cuts imposed in the $4 \ell+E_{T}$ scenario at 14,27 and $100 \mathrm{TeV}$. We give the cross-section for background and benchmark scenarios in fb. Statistical significances $\sigma_{\mathcal{A}}$ and $\sigma_{\mathcal{B}}$ of $4 \ell+E_{T}$ signal are given for each energy

\begin{tabular}{|c|c|c|c|}
\hline $4 \ell+\not_{T} @ 14 \mathrm{TeV}$ & Background [fb] & BP1 [fb] & BP3 [fb] \\
\hline No Cut & $3.45 \times 10^{-2}$ & $1.99 \times 10^{-3}$ & $1.86 \times 10^{-3}$ \\
\hline$\left|\eta_{i}\right|<2.5, \Delta R_{12} \geq 0.5$ & $2.89 \times 10^{-2}$ & $5.31 \times 10^{-4}$ & $4.63 \times 10^{-4}$ \\
\hline$p_{T}\left(\ell_{1}\right)>100 \mathrm{GeV}$ & $1.7 \times 10^{-2}$ & $5.1 \times 10^{-4}$ & $4.61 \times 10^{-4}$ \\
\hline$p_{T}\left(\ell_{2}\right)>50 \mathrm{GeV}$ & $1.58 \times 10^{-2}$ & $5.02 \times 10^{-4}$ & $4.61 \times 10^{-4}$ \\
\hline$p_{T}\left(\ell_{3}\right)>25 \mathrm{GeV}$ & $1.52 \times 10^{-2}$ & $4.87 \times 10^{-4}$ & $4.59 \times 10^{-4}$ \\
\hline$p_{T}\left(\ell_{4}\right)>15 \mathrm{GeV}$ & $1.4 \times 10^{-2}$ & $4.44 \times 10^{-4}$ & $4.31 \times 10^{-4}$ \\
\hline$E_{T}>400 \mathrm{GeV}$ & $1.0 \times 10^{-4}$ & $5.5 \times 10^{-5}$ & $1.3 \times 10^{-4}$ \\
\hline Significance: $\sigma_{\mathcal{A}}$ & $\mathcal{L}=3 \mathrm{ab}^{-1}$ & $0.24 \sigma$ & $0.46 \sigma$ \\
\hline$\sigma_{\mathcal{B}}$ & & $0.19 \sigma$ & $0.38 \sigma$ \\
\hline \multicolumn{4}{|l|}{$4 \ell+E_{T} @ 27 \mathrm{TeV}$} \\
\hline No Cut & $9.59 \times 10^{-2}$ & $3.03 \times 10^{-2}$ & $3.86 \times 10^{-2}$ \\
\hline$\left|\eta_{i}\right|<2.5, \Delta R_{12} \geq 0.5$ & $7.52 \times 10^{-2}$ & $5.9 \times 10^{-3}$ & $4.14 \times 10^{-3}$ \\
\hline$p_{T}\left(\ell_{1}\right)>100 \mathrm{GeV}$ & $4.7 \times 10^{-2}$ & $5.74 \times 10^{-3}$ & $4.13 \times 10^{-3}$ \\
\hline$p_{T}\left(\ell_{2}\right)>50 \mathrm{GeV}$ & $4.39 \times 10^{-2}$ & $5.68 \times 10^{-3}$ & $4.13 \times 10^{-3}$ \\
\hline$p_{T}\left(\ell_{3}\right)>25 \mathrm{GeV}$ & $4.2 \times 10^{-2}$ & $5.52 \times 10^{-3}$ & $4.12 \times 10^{-3}$ \\
\hline$p_{T}\left(\ell_{4}\right)>15 \mathrm{GeV}$ & $3.86 \times 10^{-2}$ & $5.08 \times 10^{-3}$ & $3.9 \times 10^{-3}$ \\
\hline$E_{T}>350 \mathrm{GeV}$ & $1.1 \times 10^{-3}$ & $1.2 \times 10^{-3}$ & $2.2 \times 10^{-3}$ \\
\hline Significance: $\sigma_{\mathcal{A}}$ & $\mathcal{L}=15 \mathrm{ab}^{-1}$ & $3.1 \sigma$ & $4.6 \sigma$ \\
\hline$\sigma_{\mathcal{B}}$ & & $2.6 \sigma$ & $4.1 \sigma$ \\
\hline \multicolumn{4}{|l|}{$4 \ell+\not E_{T} @ 100 \mathrm{TeV}$} \\
\hline No Cut & 2.48 & $9.38 \times 10^{-1}$ & 1.64 \\
\hline$\left|\eta_{i}\right|<2.5, \Delta R_{12} \geq 0.5$ & $6.56 \times 10^{-1}$ & $1.33 \times 10^{-1}$ & $1.16 \times 10^{-1}$ \\
\hline$p_{T}\left(\ell_{1}\right)>100 \mathrm{GeV}$ & $4.46 \times 10^{-1}$ & $1.3 \times 10^{-1}$ & $1.15 \times 10^{-1}$ \\
\hline$p_{T}\left(\ell_{2}\right)>50 \mathrm{GeV}$ & $4.1 \times 10^{-1}$ & $1.29 \times 10^{-1}$ & $1.14 \times 10^{-1}$ \\
\hline$p_{T}\left(\ell_{3}\right)>25 \mathrm{GeV}$ & $3.83 \times 10^{-1}$ & $1.22 \times 10^{-1}$ & $1.01 \times 10^{-1}$ \\
\hline$p_{T}\left(\ell_{4}\right)>15 \mathrm{GeV}$ & $3.1 \times 10^{-1}$ & $9.9 \times 10^{-2}$ & $8.44 \times 10^{-2}$ \\
\hline$E_{T}>800 \mathrm{GeV}$ & $7.1 \times 10^{-3}$ & $8.0 \times 10^{-3}$ & $2.3 \times 10^{-2}$ \\
\hline Significance: $\sigma_{\mathcal{A}}$ & $\mathcal{L}=30 \mathrm{ab}^{-1}$ & $11.0 \sigma$ & $23.0 \sigma$ \\
\hline$\sigma_{\mathcal{B}}$ & & $9.4 \sigma$ & $22.0 \sigma$ \\
\hline
\end{tabular}
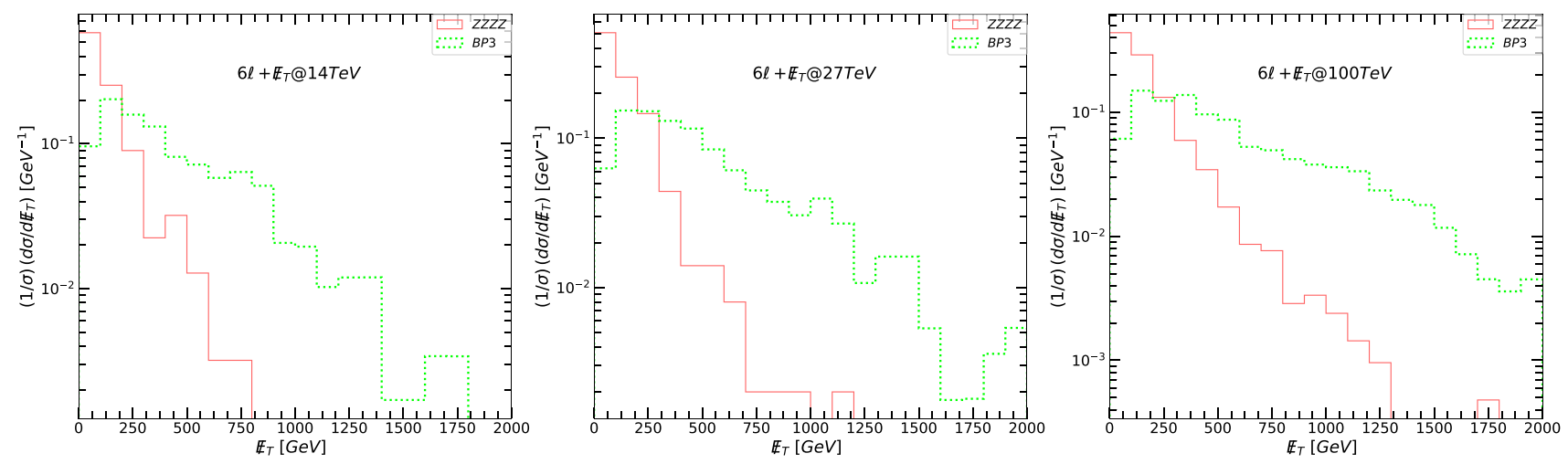

Fig. 8 The missing transverse energy $E_{T}$, for the signal and background for the $6 \ell+E_{T}$ signal. (Left-hand) signals and backgrounds at $14 \mathrm{TeV}$, (middle) at $27 \mathrm{TeV}$, and (right-hand) at $100 \mathrm{TeV}$. The main backgrounds (four-bosons) are indicated in solid lines while the dotted green line represents the signal BP3 

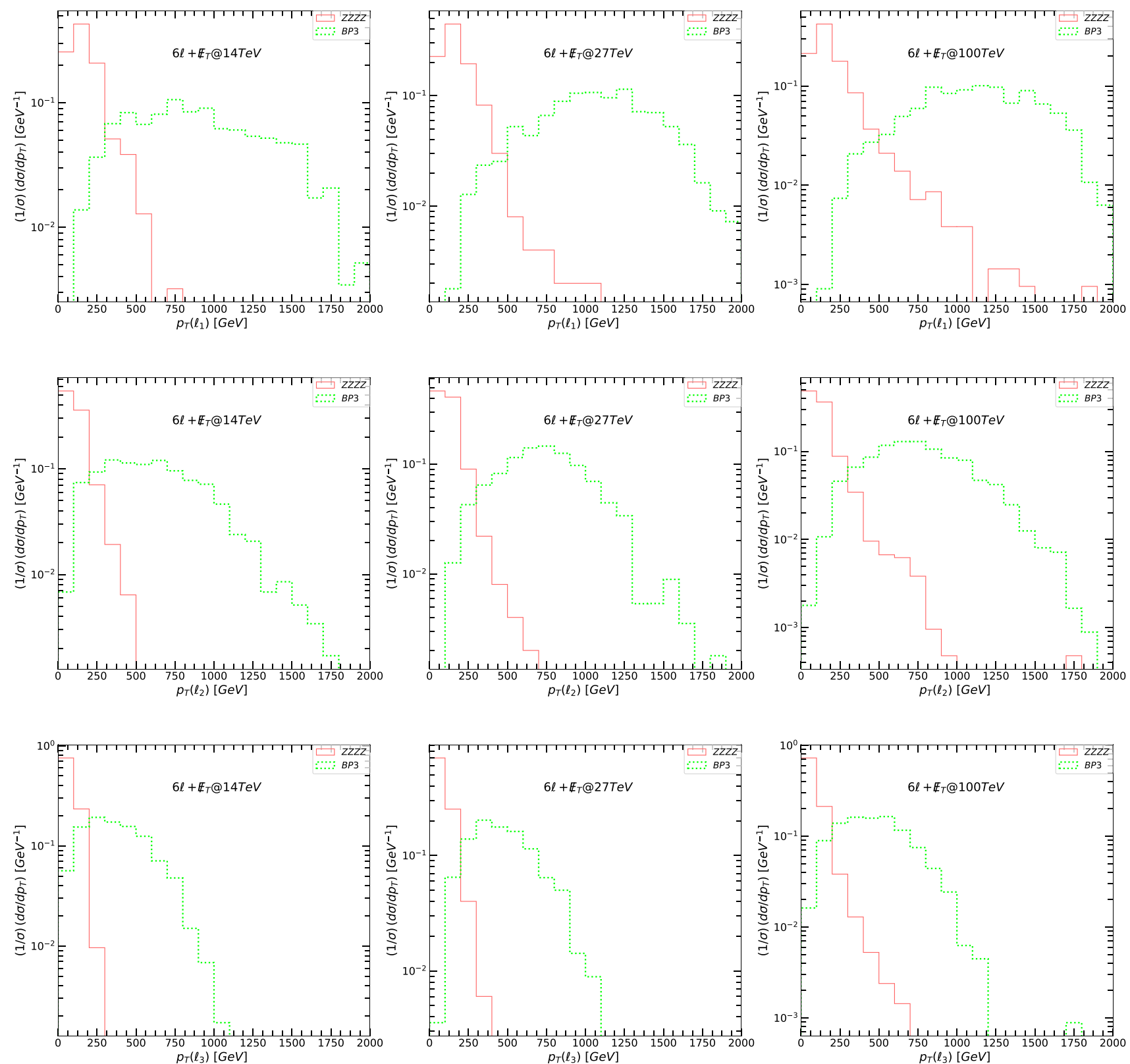

Fig. 9 The transverse momentum $p_{T}$, for the leading lepton $\ell_{1}$ (top panels), next-to-leading lepton $\ell_{2}$ (second panels), and next-to-next to leading lepton $\ell_{3}$ (bottom panels) for the signal and background for the $6 \ell+\mathbb{E}_{T}$ signal. (Left-hand) signals and backgrounds at $14 \mathrm{TeV}$, (mid- dle) at $27 \mathrm{TeV}$, and (right-hand) at $100 \mathrm{TeV}$. The main backgrounds (four-bosons) are indicated in solid lines while the dotted green line represents the signal BP3 
Table 9 Signal selection strategy and cuts imposed in the $6 \ell+\mathbb{E}_{T}$ scenario at 14, 27 and $100 \mathrm{TeV}$. We give the cross-section for background and benchmark scenarios in fb. Statistical significances $\sigma_{\mathcal{A}}$ and $\sigma_{\mathcal{B}}$ of $6 \ell+E_{T}$ signal are given for each energy

\begin{tabular}{|c|c|c|}
\hline $6 \ell+\not E_{T} @ 14 \mathrm{TeV}$ & Background [fb] & $B P 3[f b]$ \\
\hline No Cut & $3.12 \times 10^{-7}$ & $8.1 \times 10^{-5}$ \\
\hline$\left|\eta_{i}\right|<2.5, \Delta R_{12} \geq 0.2$ & $2.91 \times 10^{-7}$ & $4.37 \times 10^{-5}$ \\
\hline$\left\{\begin{array}{c}p_{T}\left(\ell_{1}\right)>50 \mathrm{GeV}, p_{T}\left(\ell_{2,3,4}\right)>20 \mathrm{GeV}, \\
p_{T}\left(\ell_{5}\right)>10 \mathrm{GeV}, p_{T}\left(\ell_{6}\right)>5 \mathrm{GeV}\end{array}\right\}$ & $2.91 \times 10^{-7}$ & $4.37 \times 10^{-5}$ \\
\hline $\mathbb{E}_{T}>100 \mathrm{GeV}$ & $1.2 \times 10^{-7}$ & $3.8 \times 10^{-5}$ \\
\hline Significance: $\sigma_{\mathcal{A}}$ & $\mathcal{L}=3 \mathrm{ab}^{-1}$ & $0.34 \sigma$ \\
\hline$\sigma_{\mathcal{B}}$ & & $0.40 \sigma$ \\
\hline \multicolumn{3}{|l|}{$6 \ell+E_{T} @ 27 T e V$} \\
\hline No Cut & $8.4 \times 10^{-7}$ & $1.71 \times 10^{-3}$ \\
\hline$\left|\eta_{i}\right|<2.5, \Delta R_{12} \geq 0.2$ & $7.87 \times 10^{-7}$ & $7.03 \times 10^{-4}$ \\
\hline$\left\{\begin{array}{c}p_{T}\left(\ell_{1}\right)>50 \mathrm{GeV}, p_{T}\left(\ell_{2,3,4}\right)>20 \mathrm{GeV}, \\
p_{T}\left(\ell_{5}\right)>10 \mathrm{GeV}, p_{T}\left(\ell_{6}\right)>5 \mathrm{GeV}\end{array}\right\}$ & $7.80 \times 10^{-7}$ & $7.03 \times 10^{-4}$ \\
\hline$\not_{T}>100 \mathrm{GeV}$ & $3.9 \times 10^{-7}$ & $6.5 \times 10^{-4}$ \\
\hline Significance: $\sigma_{\mathcal{A}}$ & $\mathcal{L}=15 \mathrm{ab}^{-1}$ & $3.1 \sigma$ \\
\hline$\sigma_{\mathcal{B}}$ & & $3.7 \sigma$ \\
\hline \multicolumn{3}{|l|}{$6 \ell+\not E_{T} @ 100 T e V$} \\
\hline No Cut & $2.27 \times 10^{-5}$ & $9.52 \times 10^{-2}$ \\
\hline$\left|\eta_{i}\right|<2.5, \Delta R_{12} \geq 0.2$ & $1.04 \times 10^{-5}$ & $4.92 \times 10^{-3}$ \\
\hline$p_{T}\left(\ell_{1}\right)>100 \mathrm{GeV}$ & $8.27 \times 10^{-6}$ & $4.92 \times 10^{-3}$ \\
\hline$p_{T}\left(\ell_{2}\right)>50 \mathrm{GeV}, p_{T}\left(\ell_{3}\right)>20 \mathrm{GeV}$ & $8.17 \times 10^{-6}$ & $4.92 \times 10^{-3}$ \\
\hline$p_{T}\left(\ell_{4}\right)>20 \mathrm{GeV}$ & $8.07 \times 10^{-6}$ & $4.66 \times 10^{-3}$ \\
\hline$p_{T}\left(\ell_{5}\right)>15 \mathrm{GeV}$ & $7.5 \times 10^{-6}$ & $3.65 \times 10^{-3}$ \\
\hline$p_{T}\left(\ell_{6}\right)>5 \mathrm{GeV}$ & $6.77 \times 10^{-6}$ & $2.88 \times 10^{-3}$ \\
\hline$E_{T}>100 \mathrm{GeV}$ & $3.9 \times 10^{-6}$ & $2.3 \times 10^{-3}$ \\
\hline Significance: $\sigma_{\mathcal{A}}$ & $\mathcal{L}=30 \mathrm{ab}^{-1}$ & $8.3 \sigma$ \\
\hline$\sigma_{\mathcal{B}}$ & & $9.7 \sigma$ \\
\hline
\end{tabular}

Acknowledgements The work of M.F. has been partly supported by NSERC through Grant number SAP105354. M.F. is grateful to a TUBITAK-BIDEB 2221 Grant which enabled this collaboration, even if the spread of COVID-19 prevented a visit to METU.

Data Availability Statement This manuscript has associated data in a data repository. [Authors' comment: All data included in this manuscript are available upon request by contacting one of the authors.]

Open Access This article is licensed under a Creative Commons Attribution 4.0 International License, which permits use, sharing, adaptation, distribution and reproduction in any medium or format, as long as you give appropriate credit to the original author(s) and the source, provide a link to the Creative Commons licence, and indicate if changes were made. The images or other third party material in this article are included in the article's Creative Commons licence, unless indicated otherwise in a credit line to the material. If material is not included in the article's Creative Commons licence and your intended use is not permitted by statutory regulation or exceeds the permitted use, you will need to obtain permission directly from the copyright holder. To view a copy of this licence, visit http://creativecomm ons.org/licenses/by/4.0/.

Funded by SCOAP ${ }^{3}$.

\section{References}

1. W. Buchmuller, D. Wyler, Nucl. Phys. B 268, 621 (1986). https:// doi.org/10.1016/0550-3213(86)90262-2

2. M. Cvetic, P. Langacker, Mod. Phys. Lett. A 11, 1247 (1996). https://doi.org/10.1142/S0217732396001260

3. D. Suematsu, Y. Yamagishi, Int. J. Mod. Phys. A 10, 4521 (1995). https://doi.org/10.1142/S0217751X95002096

4. H.S. Lee, K.T. Matchev, T.T. Wang, Phys. Rev. D 77, 015016 (2008). https://doi.org/10.1103/PhysRevD.77.015016

5. D.A. Demir, Phys. Rev. D 59, 015002 (1999). https://doi.org/10. 1103/PhysRevD.59.015002

6. S.M. Barr, Phys. Rev. Lett. 55, 2778 (1985). https://doi.org/10. 1103/PhysRevLett.55.2778

7. T.G. Rizzo, Phys. Rev. D 59, 015020 (1998). https://doi.org/10. 1103/PhysRevD.59.015020

8. J.L. Hewett, T.G. Rizzo, Phys. Rep. 183, 193 (1989). https://doi. org/10.1016/0370-1573(89)90071-9

9. P. Athron, D. Harries, R. Nevzorov, A.G. Williams, JHEP 12, 128 (2016). https://doi.org/10.1007/JHEP12(2016)128

10. P. Langacker, J. Wang, Phys. Rev. D 58, 115010 (1998). https:// doi.org/10.1103/PhysRevD.58.115010 
11. G. Cleaver, M. Cvetic, J.R. Espinosa, L.L. Everett, P. Langacker, Phys. Rev. D 57, 2701 (1998). https://doi.org/10.1103/PhysRevD. 57.2701

12. F. Del Aguila, Acta Phys. Pol. B 25, 1317 (1994)

13. A. Leike, Phys. Rep. 317, 143 (1999). https://doi.org/10.1016/ S0370-1573(98)00133-1

14. K.S. Babu, C.F. Kolda, J. March-Russell, Phys. Rev. D 57, 6788 (1998). https://doi.org/10.1103/PhysRevD.57.6788

15. P. Langacker, Rev. Mod. Phys. 81, 1199 (2009). https://doi.org/ 10.1103/RevModPhys.81.1199

16. J. Erler, P. Langacker, S. Munir, E. Rojas, JHEP 08, 017 (2009). https://doi.org/10.1088/1126-6708/2009/08/017

17. J. Erler, P. Langacker, S. Munir, E. Rojas, JHEP 11, 076 (2011). https://doi.org/10.1007/JHEP11(2011)076

18. R. Diener, S. Godfrey, T.A.W. Martin, in Particles and fields. Proceedings, Meeting of the Division of the American Physical Society, DPF 2009, Detroit, USA, July 26-31, 2009 (2009)

19. E. Accomando, C. Coriano, L. Delle Rose, J. Fiaschi, C. Marzo, S. Moretti, EPJ Web Conf. 129, 00006 (2016). https://doi.org/10. 1051/epjconf/201612900006

20. T.G. Rizzo, Theoretical advanced study institute in elementary particle physics: exploring new frontiers using colliders and neutrinos 537-575 (2006)

21. J. Batley et al., Phys. Lett. B 746, 178 (2015). https://doi.org/10. 1016/j.physletb.2015.04.068

22. H. Merkel et al., Phys. Rev. Lett. 112(22), 221802 (2014). https:// doi.org/10.1103/PhysRevLett.112.221802

23. A. Anastasi et al., Phys. Lett. B 757, 356 (2016). https://doi.org/ 10.1016/j.physletb.2016.04.019

24. F. Archilli et al., Phys. Lett. B 706, 251 (2012). https://doi.org/ 10.1016/j.physletb.2011.11.033

25. D. Babusci et al., Phys. Lett. B 736, 459 (2014). https://doi.org/ 10.1016/j.physletb.2014.08.005

26. R. Aaij et al., Phys. Rev. Lett. 120(6), 061801 (2018). https://doi. org/10.1103/PhysRevLett.120.061801

27. A.M. Sirunyan et al., JHEP 02, 074 (2019). https://doi.org/10. 1007/JHEP02(2019)074

28. B. Batell, R. Essig, Z. Surujon, Phys. Rev. Lett. 113(17), 171802 (2014). https://doi.org/10.1103/PhysRevLett.113.171802

29. L. Marsicano, M. Battaglieri, M. Bondi', C.R. Carvajal, A. Celentano, M. De Napoli, R. De Vita, E. Nardi, M. Raggi, P. Valente, Phys. Rev. D 98(1), 015031 (2018). https://doi.org/10. 1103/PhysRevD.98.015031

30. S. Gninenko, Phys. Lett. B 713, 244 (2012). https://doi.org/10. 1016/j.physletb.2012.06.002

31. W. Altmannshofer et al., PTEP 2019(12), $123 \mathrm{C} 01$ (2019). https:// doi.org/10.1093/ptep/ptz106. (Erratum: PTEP 2020, 029201 (2020))

32. P. Ilten, Y. Soreq, J. Thaler, M. Williams, W. Xue, Phys. Rev. Lett. 116(25), 251803 (2016). https://doi.org/10.1103/PhysRevLett. 116.251803

33. P. Ilten, J. Thaler, M. Williams, W. Xue, Phys. Rev. D 92(11), 115017 (2015). https://doi.org/10.1103/PhysRevD.92.115017

34. R. Aaij, et al., LHCB-PUB-2018-009, CERN-LHCC-2018-027 (2018)

35. J.L. Feng, I. Galon, F. Kling, S. Trojanowski, Phys. Rev. D 97(3), 035001 (2018). https://doi.org/10.1103/PhysRevD.97.035001

36. P. Adrian et al., Phys. Rev. D 98(9), 091101 (2018). https://doi. org/10.1103/PhysRevD.98.091101

37. A. Berlin, S. Gori, P. Schuster, N. Toro, Phys. Rev. D 98(3), 035011 (2018). https://doi.org/10.1103/PhysRevD.98.035011

38. M. Karliner, M. Low, J.L. Rosner, L.T. Wang, Phys. Rev. D 92(3), 035010 (2015). https://doi.org/10.1103/PhysRevD.92.035010

39. D. Curtin, R. Essig, S. Gori, J. Shelton, JHEP 02, 157 (2015). https://doi.org/10.1007/JHEP02(2015)157
40. M. D'Onofrio, O. Fischer, Z.S. Wang, Phys. Rev. D 101(1), 015020 (2020). https://doi.org/10.1103/PhysRevD.101.015020

41. S. Bilmis, I. Turan, T. Aliev, M. Deniz, L. Singh, H. Wong, Phys. Rev. D 92(3), 033009 (2015). https://doi.org/10.1103/PhysRevD. 92.033009

42. D.A. Demir, G.L. Kane, T.T. Wang, Phys. Rev. D 72, 015012 (2005). https://doi.org/10.1103/PhysRevD.72.015012

43. O.C. Anoka, K.S. Babu, I. Gogoladze, Nucl. Phys. B 687, 3 (2004). https://doi.org/10.1016/j.nuclphysb.2004.03.009

44. G. Corcella, Eur. Phys. J. C 75(6), 264 (2015). https://doi.org/10. 1140/epjc/s10052-015-3459-9

45. S. Corcella, S. Gentile, Nucl. Phys. B 866, 293 (2013). https://doi. org/10.1016/j.nuclphysb.2012.09.009. (Erratum: Nucl. Phys. B 868, 554-555 (2013))

46. G. Corcella, in 18th International Conference From the Planck Scale to the Electroweak Scale (Planck 2015) Ioannina, Greece, May 25-29, 2015 (2015). arXiv: 1510.02412

47. G. Corcella, EPJ Web Conf. 60, 18011 (2013). https://doi.org/10. 1051/epjconf/20136018011

48. T. Gherghetta, T.A. Kaeding, G.L. Kane, Phys. Rev. D 57, 3178 (1998). https://doi.org/10.1103/PhysRevD.57.3178

49. C.F. Chang, K. Cheung, T.C. Yuan, JHEP 09, 058 (2011). https:// doi.org/10.1007/JHEP09(2011)058

50. M. Baumgart, T. Hartman, C. Kilic, L.T. Wang, JHEP 11, 084 (2007). https://doi.org/10.1088/1126-6708/2007/11/084

51. J. Kang, P. Langacker, Phys. Rev. D 71, 035014 (2005). https:// doi.org/10.1103/PhysRevD.71.035014

52. P. Langacker, G. Paz, L.T. Wang, I. Yavin, Phys. Rev. D 77, 085033 (2008). https://doi.org/10.1103/PhysRevD.77.085033

53. B. Bhattacherjee, N. Khan, A. Patra, Phys. Rev. D 100(7), 075010 (2019). https://doi.org/10.1103/PhysRevD.100.075010

54. G. Aad, et al., CERN-EP-2019-030 (2019)

55. M. Aaboud et al., Phys. Rev. D 96(5), 052004 (2017). https://doi. org/10.1103/PhysRevD.96.052004

56. A.M. Sirunyan et al., JHEP 08, 130 (2018). https://doi.org/10. 1007/JHEP08(2018)130

57. M. Aaboud et al., JHEP 01, 055 (2018). https://doi.org/10.1007/ JHEP01(2018)055

58. M. Aaboud, et al., CERN-EP-2018-350 (2019)

59. J. Erler, P. Langacker, T. Li, Phys. Rev. D 66, 015002 (2002). https://doi.org/10.1103/PhysRevD.66.015002

60. M. Frank, L. Selbuz, I. Turan, Eur. Phys. J. C 73, 2656 (2013). https://doi.org/10.1140/epjc/s10052-013-2656-7

61. K.S. Babu, C.F. Kolda, J. March-Russell, Phys. Rev. D 54, 4635 (1996). https://doi.org/10.1103/PhysRevD.54.4635

62. C.W. Chiang, T. Nomura, K. Yagyu, JHEP 05, 106 (2014). https:// doi.org/10.1007/JHEP05(2014)106

63. J.Y. Araz, G. Corcella, M. Frank, B. Fuks, JHEP 02, 092 (2018). https://doi.org/10.1007/JHEP02(2018)092

64. G.H. Duan, X. Fan, M. Frank, C. Han, J.M. Yang, Phys. Lett. B 789, 54 (2019). https://doi.org/10.1016/j.physletb.2018.12.005

65. M. Frank, K. Huitu, S. Mondal, Phys. Rev. D 100(11), 115018 (2019). https://doi.org/10.1103/PhysRevD.100.115018

66. E. Accomando, D. Becciolini, A. Belyaev, S. Moretti, C. Shepherd-Themistocleous, JHEP 10, 153 (2013). https://doi.org/ 10.1007/JHEP10(2013)153

67. E. Accomando, F. Coradeschi, T. Cridge, J. Fiaschi, F. Hautmann, S. Moretti, C. Shepherd-Themistocleous, C. Voisey, Phys. Lett. B 803, 135293 (2020). https://doi.org/10.1016/j.physletb.2020. 135293

68. M. Frank, Y. Hiçyılmaz, S. Moretti, O. Özdal, JHEP 05, 123 (2020). https://doi.org/10.1007/JHEP05(2020)123

69. P. Langacker, Phys. Rev. D 58, 093017 (1998). https://doi.org/10. 1103/PhysRevD.58.093017

70. D.A. Demir, M. Frank, L. Selbuz, I. Turan, Phys. Rev. D 83, 095001 (2011). https://doi.org/10.1103/PhysRevD.83.095001 
71. P. Bechtle, O. Brein, S. Heinemeyer, O. Stål, T. Stefaniak, G. Weiglein, K.E. Williams, Eur. Phys. J. C 74(3), 2693 (2014). https:// doi.org/10.1140/epjc/s10052-013-2693-2

72. P. Bechtle, S. Heinemeyer, O. Stål, T. Stefaniak, G. Weiglein, Eur. Phys. J. C 74(2), 2711 (2014). https://doi.org/10.1140/epjc/ s10052-013-2711-4

73. R. Aaij et al., Phys. Rev. Lett. 110(2), 021801 (2013). https://doi. org/10.1103/PhysRevLett.110.021801

74. X. Cid Vidal, et al., Report from Working Group 3: Beyond the Standard Model physics at the HL-LHC and HE-LHC (2019), vol. 7, pp. 585-865. 10.23731/CYRM-2019-007.585

75. D.N. Spergel et al., Astrophys. J. Suppl. 170, 377 (2007). https:// doi.org/10.1086/513700

76. M. Tanabashi et al., Phys. Rev. D 98(3), 030001 (2018). https:// doi.org/10.1103/PhysRevD.98.030001

77. A. Belyaev, N.D. Christensen, A. Pukhov, Comput. Phys. Commun. 184, 1729 (2013). https://doi.org/10.1016/j.cpc.2013.01. 014

78. G. Bélanger, F. Boudjema, A. Goudelis, A. Pukhov, B. Zaldivar, Comput. Phys. Commun. 231, 173 (2018). https://doi.org/10. 1016/j.cpc.2018.04.027

79. T. Sjostrand, S. Mrenna, P.Z. Skands, JHEP 05, 026 (2006). https://doi.org/10.1088/1126-6708/2006/05/026

80. T. Sjostrand, S. Ask, J.R. Christiansen, R. Corke, N. Desai, P. Ilten, S. Mrenna, S. Prestel, C.O. Rasmussen, P.Z. Skands, Comput. Phys. Commun. 191, 159 (2015). https://doi.org/10.1016/j.cpc. 2015.01.024

81. J. de Favereau, C. Delaere, P. Demin, A. Giammanco, V. Le Maitre, A. Mertens, M. Selvaggi, JHEP 02, 057 (2014). https:// doi.org/10.1007/JHEP02(2014)057

82. M. Selvaggi, J. Phys. Conf. Ser. 523, 012033 (2014). https://doi. org/10.1088/1742-6596/523/1/012033

83. A. Mertens, J. Phys. Conf. Ser. 608(1), 012045 (2015). https:// doi.org/10.1088/1742-6596/608/1/012045

84. E. Conte, B. Fuks, G. Serret, Comput. Phys. Commun. 184, 222 (2013). https://doi.org/10.1016/j.cpc.2012.09.009

85. G. Aad et al., JHEP 10, 134 (2015). https://doi.org/10.1007/ JHEP10(2015) 134

86. S. Caron, J.S. Kim, K. Rolbiecki, R. Ruiz de Austri, B. Stienen, Eur. Phys. J. C 77(4), 257 (2017). https://doi.org/10.1140/epjc/ s10052-017-4814-9

87. S. Laporta, Phys. Lett. B 772, 232 (2017). https://doi.org/10.1016/ j.physletb.2017.06.056

88. T. Aoyama, T. Kinoshita, M. Nio, Phys. Rev. D 97(3), 036001 (2018). https://doi.org/10.1103/PhysRevD.97.036001

89. T. Aoyama, M. Hayakawa, T. Kinoshita, M. Nio, Phys. Rev. Lett. 109, 111807 (2012). https://doi.org/10.1103/PhysRevLett. 109.111807

90. G. Bennett et al., Phys. Rev. D 73, 072003 (2006). https://doi.org/ 10.1103/PhysRevD.73.072003

91. T. Aoyama, M. Hayakawa, T. Kinoshita, M. Nio, Phys. Rev. Lett. 109, 111808 (2012). https://doi.org/10.1103/PhysRevLett. 109.111808

92. X. Chen, Y. Wu, JHEP 10, 089 (2019). https://doi.org/10.1007/ JHEP10(2019)089

93. F. Sala, Nuovo Cim. C 39(4), 323 (2017). https://doi.org/10.1393/ ncc/i2016-16323-9
94. A.J. Buras, J.M. Gérard, W.A. Bardeen, Eur. Phys. J. C 74, 2871 (2014). https://doi.org/10.1140/epjc/s10052-014-2871-x

95. F. Staub, Comput. Phys. Commun. 185, 1773 (2014). https://doi. org/10.1016/j.cpc.2014.02.018

96. F. Staub, Adv. High Energy Phys. 2015, 840780 (2015). https:// doi.org/10.1155/2015/840780

97. W. Porod, Comput. Phys. Commun. 153, 275 (2003). https://doi. org/10.1016/S0010-4655(03)00222-4

98. W. Porod, F. Staub, Comput. Phys. Commun. 183, 2458 (2012). https://doi.org/10.1016/j.cpc.2012.05.021

99. A. Czarnecki, W.J. Marciano, Nucl. Phys. B Proc. Suppl. 76, 245 (1999). https://doi.org/10.1016/S0920-5632(99)00474-0

100. V. Barger, C. Kao, P. Langacker, H.S. Lee, Phys. Lett. B 614, 67 (2005). https://doi.org/10.1016/j.physletb.2005.03.042

101. G. Aad et al., JHEP 03, 145 (2020). https://doi.org/10.1007/ JHEP03(2020) 145

102. A.M. Sirunyan, et al., CMS-PAS-EXO-19-012 (2019)

103. M. Aaboud et al., Phys. Rev. Lett. 121(8), 081801 (2018). https:// doi.org/10.1103/PhysRevLett.121.081801

104. M. Aaboud et al., Phys. Rev. D 98, 032016 (2018). https://doi. org/10.1103/PhysRevD.98.032016

105. A.M. Sirunyan et al., JHEP 04, 031 (2019). https://doi.org/10. 1007/JHEP04(2019)031

106. M. Aaboud et al., Eur. Phys. J. C 78(7), 565 (2018). https://doi. org/10.1140/epjc/s10052-018-5995-6

107. A.M. Sirunyan, et al., CMS-PAS-EXO-19-019 (2019)

108. V. Khachatryan et al., JHEP 02, 048 (2017). https://doi.org/10. 1007/JHEP02(2017)048

109. M. Aaboud et al., JHEP 03, 042 (2018). https://doi.org/10.1007/ JHEP03(2018)042

110. A.M. Sirunyan et al., Phys. Lett. B 798, 134952 (2019). https:// doi.org/10.1016/j.physletb.2019.134952

111. M. Dobbs, J.B. Hansen, Comput. Phys. Commun. 134, 41 (2001). https://doi.org/10.1016/S0010-4655(00)00189-2

112. J. Pumplin, D. Stump, J. Huston, H. Lai, P.M. Nadolsky, W. Tung, JHEP 07, 012 (2002). https://doi.org/10.1088/1126-6708/2002/ $07 / 012$

113. J. Butterworth et al., J. Phys. G 43, 023001 (2016). https://doi. org/10.1088/0954-3899/43/2/023001

114. E. Arganda, V. Martin-Lozano, A.D. Medina, N. Mileo, JHEP 09, 056 (2018). https://doi.org/10.1007/JHEP09(2018)056

115. W. Abdallah, J. Fiaschi, S. Khalil, S. Moretti, JHEP 02, 157 (2016). https://doi.org/10.1007/JHEP02(2016)157

116. G. Cowan, K. Cranmer, E. Gross, O. Vitells, Eur. Phys. J. C 71, 15541554 (2011). https://doi.org/10.1140/epjc/ s10052-011-1554-0. (Erratum: Eur. Phys. J. C 73, 2501 (2013))

117. S. Chatrchyan et al., Eur. Phys. J. C 73(3), 2339 (2013). https:// doi.org/10.1140/epjc/s10052-013-2339-4

118. M. Aaboud et al., Phys. Rev. D 98, 012003 (2018). https://doi. org/10.1103/PhysRevD.98.012003

119. A.M. Sirunyan et al., JHEP 06, 128 (2018). https://doi.org/10. 1007/JHEP06(2018)128

120. J.Y. Araz, M. Frank, B. Fuks, Phys. Rev. D 96(1), 015017 (2017). https://doi.org/10.1103/PhysRevD.96.015017 